\title{
1999 Annual Report on Waste Generation and Pollution Prevention Progress as Required by DOE Order 5400.1
}

Prepared for the U.S. Department of Energy

Assistant Secretary for Environmental Management

Project Hanford Management Contractor for the

U.S. Department of Energy under Contract DE-AC06-96RL13200

Fluor Hanford

P.O. Box 1000

Richland, Washington

\section{RECORD COPY}




\section{Annual Report on Waste} Generation and Pollution Prevention Progress as Required by DOE Order 5400.1

P. Segall

Fluor Hanford

\section{Date Published}

March 2000

Prepared for the U.S. Department of Energy

Assistant Secretary for Environmental Management

Project Hantord Management Contractor for the

U.S. Department of Energy under Contract DE-AC06-96RL13200

Fis. Department of Energy under Contract

P.o. Box 1000

Richland, Washington

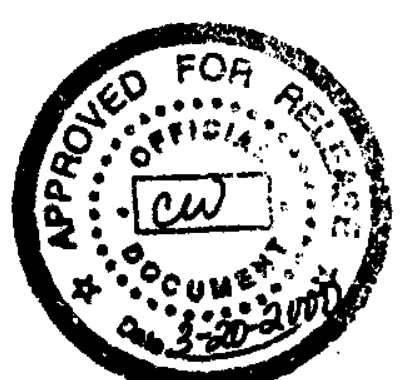

Release Stamp 


\section{LEGAL DISCLAMEA}

This report was prepared as an account of work sponsored by an agency of the United States Government. Neither thi United States Govemment nor any agency thereof, nor any of their employees, nor any of their contractors, subcontractors o their employees, makes any warranty, express or implied, or assumes any legal liability or responsibility for the accuracy, completeness, or any third party's use or the results of such use of any information, apparatus, product, or process disclosed, or represents that its use would not infringe privately owned rights. Reterence herein to any specific commercial product, process, or service by trade name, trademark, manufacturer, or otherwise, does not necessarily constitute or imply its endorsement, recommendation, or favoring by the United States Govemment or any agency thereof of th contractors or subcontractors. The views and opinions of authors expressed herein do not necessarily state or reflect those of the United States Government or any agency thereof.

This report has been reproduced from the best available copy. Avallable in paper copy and microfiche.

Available electronically at htto://wmw.doe.gov/bridge. Available for processing fee to the U.S. Department of Energy and its contractors, in paper, from:

U.S. Department of Energy

Office of Scientific and Technical Information

P.O. Box 62

Oak Fidge, TN 37831-0062

phone: 865-576-8401

fax: 865-576-5728

omail: reports Q adonis.osti.gov(423) 576-8401

Avallable for sale to the public, in paper, from:

U.S. Department of Commerce

National Technical Information Service

5285 Port Royal Road

Springtield, VA 22161

phone: $800-553-6847$

fax: 703-605-6900

email: orders e nts.fectworld.gov

online ordering:

http://uww.ntis.gov/ordering.htm

Printed in the Unted Statee of America

Total Pages: 


\section{RELEASE AUTHORIZATION}

Document HNF-6039, Rev. 0

Number:

Document Title: 1999 Annual Report on Waste Generation and Pollution Prevention

Progress as Required by DOE Order 5400.1

This document, reviewed in accordance with DOE Order 241.1, "Scientific and Technical Information Management," and DOE G 241.1-1, "Guide to the Management of Scientific and Technical Information," does not contain classified or sensitive unclassified information and is:

\section{APPROVED FOR PUBLIC RELEASE}

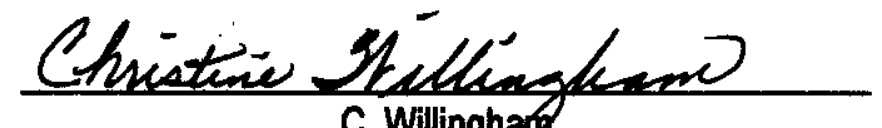

C. Willingharp

Lockheed Martin Services, Inc.

Document Control/nformation Clearance

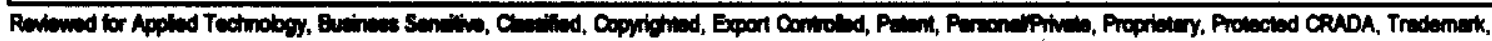

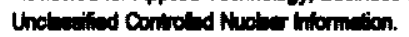

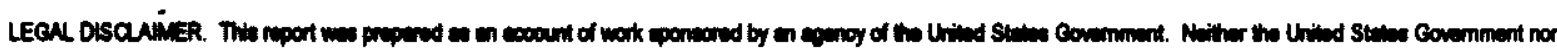

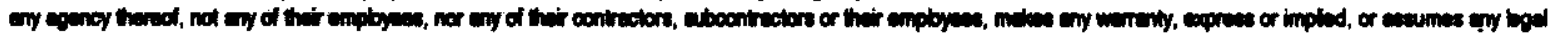

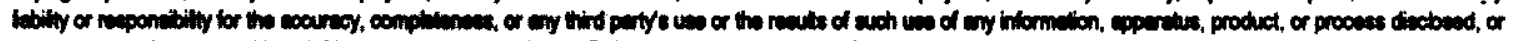

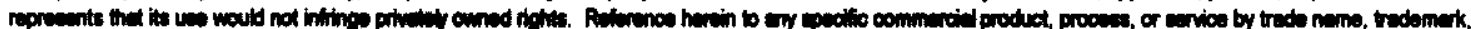

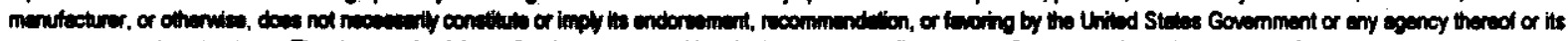

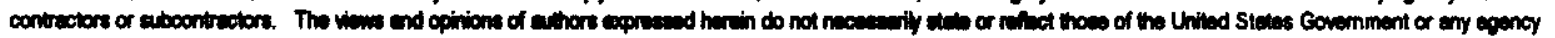

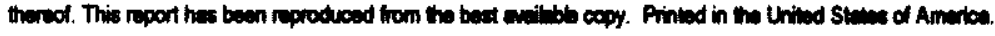


Standard Data Report

Hanford Site

Signature Page

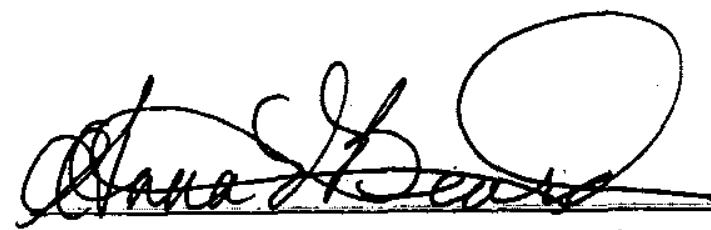

A. V. Beard, Program Manager, Richland Operations Office, DOE-RL

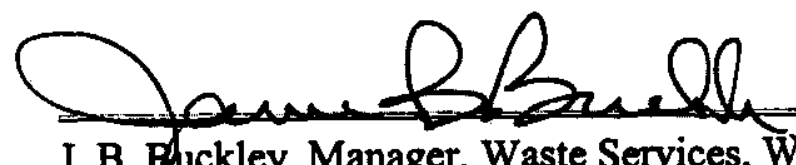

J. B. Buckley, Manager, Waste Services, W\&g e Mangement Project; Fluor Hanford
$2 / 28 / 00$

Date

$2 / 25 / 00$

Date 


\title{
Standard Data Report
}

1999 Annual Report on Waste Generation and Pollution Prevention Progress as required by DOE Order 5400.1

\author{
Hanford Site
}

Operations Office: RL

Prepared for: DOE

Prepared by: Peter Segall 


\title{
Standard Data Report
}

\author{
Hanford Site \\ General Site Information
}

Site Information:

Site Name: Hanford Site

Internet Email Address: anna_v_beard@rl.gov

Operations Office Name: RL

Lead PSO: EM

DOE Point of Contact Information:

DOE Point of Contact: Anna V. Beard

DOE Phone \#: (509)376-7472

DOE Fax \#: (509)376-4963

DOE Employee address: Address: Department of Energy, Richland Office P.O.

Contractor Point of Contact Information:

Contractor Point of Contact: Peter Segall

Contractor Phone \#: (509)372-0469

Contractor Fax \#: (509)373-0743

Contractor Address: Contractor Address: Fluor Hanford P.O. Box 1000, 


\title{
Standard Data Report
}

\author{
Hanford Site
}

General Site Information

Hanford's missions are to safely clean-up and manage the site's legacy wastes, and to develop and deploy science and technology. Through these missions Hanford will contribute to economic diversification of the region.

Hanford's environmental management or clean-up mission is to protect the health and safety of the public, workers, and the environment; control hazardous materials; and utilize the assets (people, infrastructure, and site) for other missions. Hanford's science and technology mission is to develop and deploy science and technology in the service of the nation including stewardship of the Hanford Site. Pollution Prevention is a key to the success of these missions by reducing the amount of waste to be managed and identifying/implementing cost effective waste reduction projects.

Hanford's original mission, the production of nuclear materials for the nation's defense programs, lasted more than 40 years, and like most manufacturing operations, Hanford's operations generated large quantities of waste and pollution. However, the by-products from Hanford operations pose unique problems like radiation hazards, vast volumes of contaminated water and soil, and many contaminated structures including reactors, chemical plants and evaporation ponds.

The clean-up activity is an immense and challenging undertaking. Including characterization and decommissioning of 149 single shell storage tanks, treating 28 double shell tanks, safely disposing of over 2,100 metric tons of spent nuclear fuel stored on site, removing numerous structures, and dealing with significant solid waste, ground water, and land restoration issues. 


\section{Standard Data Report}

\section{Hanford Site}

Site-Wide Recycling Activities

\begin{tabular}{|c|c|c|}
\hline Recycle Category & Quantity & \\
\hline \multicolumn{3}{|l|}{ Paper Products: } \\
\hline Office and Mixed Paper & 394.35 & $\mathrm{mt}$ \\
\hline Corrugated cardboard & 51.27 & $\mathrm{mt}$ \\
\hline Phone Books & 12.01 & $\mathrm{mt}$ \\
\hline Newpapers/Magazines & 18.42 & $\mathrm{mt}$ \\
\hline Aluminum Cans & 0.00 & $\mathrm{mt}$ \\
\hline Glass & 1.10 & $\mathrm{mt}$ \\
\hline Plastic & 0.79 & $\mathrm{mt}$ \\
\hline Styrofoam & 0.00 & $\mathrm{mt}$ \\
\hline \multicolumn{3}{|l|}{ Scrap Metals: } \\
\hline Stainless steel & 0.00 & $\mathrm{mt}$ \\
\hline Copper & 0.00 & $\mathrm{mt}$ \\
\hline Iron & 499.51 & $\mathrm{mt}$ \\
\hline Aluminum & 0.00 & $\mathrm{mt}$ \\
\hline Lead & 29.90 & $\mathrm{mt}$ \\
\hline Zinc & 0.00 & $\mathrm{mt}$ \\
\hline Other: (see discussion below) & 70.32 & $\mathrm{mt}$ \\
\hline \multicolumn{3}{|l|}{ Precious metals: } \\
\hline Silver & 0.00 & $\mathrm{mt}$ \\
\hline Gold & 0.00 & $\mathrm{mt}$ \\
\hline Platinum & 0.00 & $\mathrm{mt}$ \\
\hline Other: (see discussion below) & 0.00 & $\mathrm{mt}$ \\
\hline Toner cartridges & 13.18 & $\mathrm{mt}$ \\
\hline Batteries & 39.69 & $\mathrm{mt}$ \\
\hline Engine oils & 34.04 & $\mathrm{mt}$ \\
\hline Tires & 11.70 & $\mathrm{mt}$ \\
\hline Food waste & 0.00 & $\mathrm{mt}$ \\
\hline Concrete & 0.00 & $\mathrm{mt}$ \\
\hline Wood (chips, compost) & 131.90 & $\mathrm{mt}$ \\
\hline Other: (see discussion below) & 163.87 & $\mathrm{mt}$ \\
\hline
\end{tabular}




\section{Explanation of "Other" recycling amounts:}

Explanation for "other" amounts: The scrap metal "other" is non-ferrous metals consisting of stainless steel, copper, brass, aluminum, zinc, and tin. Additional sanitary waste recycling activities noted under "other" are discussed in the Hanford CY 1999 Accomplishments. These recycling activities include items such as software $(14.74 \mathrm{mt})$, concrete crusher $(77.00 \mathrm{mt})$, propylene glycol $(4.35 \mathrm{mt})$, excess furniture (33.04 mt), intact and crushed fluorescent tubes $(10.20 \mathrm{mt})$, non-PCB light ballasts (4.36 $\mathrm{mt})$, DOW $21 \mathrm{Resin}(5.00 \mathrm{mt})$, antifreeze $(5.10 \mathrm{mt})$, compressed gas tanks $(5.40 \mathrm{mt})$, and other miscellaneous items $(5.38 \mathrm{mt})$.

\section{Sanitary Waste}

\begin{tabular}{|l|l|l|}
\hline Routine & Cleanup/Stabilization \\
\hline 760.79 & $\mathrm{mt}$ & $\mathbf{3 9 0 . 5 2}$ \\
\hline
\end{tabular}




\section{Standard Data Report}

\section{Hanford Site}

Total Site Generation - Routine vs. Cleanup/Stabilization Waste

\begin{tabular}{|c|c|c|c|c|c|c|}
\hline Waste Type & \begin{tabular}{|c|}
$\begin{array}{c}\text { Routine } \\
\text { Waste }\end{array}$ \\
\end{tabular} & Unit & $\begin{array}{c}\text { Cleanup/Stabilization } \\
\text { Waste }\end{array}$ & Unit & Total Waste & Uni \\
\hline $\begin{array}{l}\text { High Level Waste } \\
\text { (Liquid) }\end{array}$ & 0.00 & $\mathrm{~m} 3$ & 0.00 & $\mathrm{~m} 3$ & 0.00 & $m^{3}$ \\
\hline High Level Waste (Solid) & 0.00 & $\mathrm{~m} 3$ & 0.00 & $\mathrm{~m} 3$ & 0.00 & $\mathrm{~m} 3$ \\
\hline $\begin{array}{l}\text { Transuranic Waste } \\
\text { (Liquid) }\end{array}$ & 0.00 & m3 & 0.00 & $\mathrm{~m} 3$ & 0.00 & $\mathrm{~m}^{3}$ \\
\hline Transuranic Waste (Solid) & 0.00 & $\mathrm{~m} 3$ & 11.99 & $\mathrm{~m} 3$ & 11.99 & $\mathrm{~m} 3$ \\
\hline $\begin{array}{l}\text { Mixed Transuranic Waste } \\
\text { (Liquid) }\end{array}$ & 0.00 & $\mathrm{~m} 3$ & 0.00 & $\mathrm{~m} 3$ & 0.00 & $\| m^{2}$ \\
\hline \begin{tabular}{|l|} 
Mixed Transuranic Waste \\
(Solid)
\end{tabular} & 0.00 & $\mathrm{~m} 3$ & $20.47 \|$ & $\mathrm{m} 3$ & 20.47 & $\mathrm{~m}^{3}$ \\
\hline Low Level Waste (Liquid) & 0.00 & $\mathrm{~m} 3$ & 0.00 & $\mathrm{~m} 3$ & 0.00 & $\mathrm{~m} 3$ \\
\hline Low Level Waste (Solid) & 370.61 & $\mathrm{~m} 3$ & $289,345.00$ & $\mathrm{~m} 3$ & $289,715.60$ & $\mathrm{~m} 3$ \\
\hline $\begin{array}{l}\text { Mixed Low Level Waste } \\
\text { (Liquid) }\end{array}$ & 0.00 & $\mathrm{~m} 3$ & $0.00 \|$ & $\mathrm{m} 3$ & 0.00 & \\
\hline $\begin{array}{l}\text { Mixed Low Level Waste } \\
\text { (Solid) }\end{array}$ & 96.77 & $\mathrm{~m} 3$ & 363.44 & $\mathrm{~m} 3$ & 460.21 & $\mathrm{~m} 3$ \\
\hline RCRA Regluated & 4.88 & $\mathrm{mt}$ & 94.73 & $\mathrm{mt}$ & 99.61 & $\mathrm{mt}$ \\
\hline State Regulated & 0.67 & $\mathrm{mt}$ & 13.02 & $\mathrm{mt}$ & 13.69 & $\mathrm{mt}$ \\
\hline TSCA Regulated & 0.00 & $\mathrm{mt}$ & 11.06 & $\mathrm{mt}$ & 11.06 & it \\
\hline Mixed TSCA & 0.00 & $\mathrm{mt}$ & 4.67 & $\mathrm{mt}$ & 4.67 & $\mathrm{mt}$ \\
\hline
\end{tabular}




\section{Standard Data Report}

\section{Hanford Site}

Site Waste Generation by PSO

PSO: EM

\begin{tabular}{|c|c|c|c|c|c|c|}
\hline Waste Type & $\begin{array}{c}\text { Routine } \\
\text { Waste }\end{array}$ & Unit & $\begin{array}{c}\text { Cleanup/Stabilization } \\
\text { Waste }\end{array}$ & Unit & Total Waste & Unit \\
\hline $\begin{array}{l}\text { High Level Waste } \\
\text { (Liquid) }\end{array}$ & 0.00 & $\mathrm{~m} 3$ & 0.00 & $\mathrm{~m} 3$ & 0.00 & $\mathrm{~m} 3$ \\
\hline High Level Waste (Solid) & 0.00 & $\mathrm{~m} 3$ & 0.00 & $\mathrm{~m} 3$ & 0.00 & $\mathrm{~m} 3$ \\
\hline $\begin{array}{l}\text { Transuranic Waste } \\
\text { (Liquid) }\end{array}$ & 0.00 & $\mathrm{~m} 3$ & 0.00 & $\mathrm{~m} 3$ & 0.00 & $\mathrm{~m} 3$ \\
\hline Transuranic Waste (Solid) & 0.00 & $\mathrm{~m} 3$ & 11.99 & $\mathrm{~m} 3$ & 11.99 & $\mathrm{~m} 3$ \\
\hline $\begin{array}{l}\text { Mixed Transuranic Waste } \\
\text { (Liquid) }\end{array}$ & 0.00 & $\mathrm{~m} 3$ & 0.00 & $\mathrm{~m} 3$ & 0.00 & $\mathrm{~m} 3$ \\
\hline $\begin{array}{l}\text { Mixed Transuranic Waste } \\
\text { (Solid) }\end{array}$ & 0.00 & $\mathrm{~m} 3$ & 20.47 & $\mathrm{~m} 3$ & 20.47 & $\mathrm{~m} 3$ \\
\hline Low Level Waste (Liquid) & 0.00 & $\mathrm{~m} 3$ & 0.00 & $\mathrm{~m} 3$ & 0.00 & $\mathrm{~m} 3$ \\
\hline Low Level Waste (Solid) & 370.61 & $\mathrm{~m} 3$ & $289,345.00$ & $\mathrm{~m} 3$ & $289,715.60$ & $\mathrm{~m} 3$ \\
\hline $\begin{array}{l}\text { Mixed Low Level Waste } \\
\text { (Liquid) }\end{array}$ & 0.00 & $\mathrm{~m} 3$ & 0.00 & $\mathrm{~m} 3$ & 0.00 & m3 \\
\hline $\begin{array}{l}\text { Mixed Low Level Waste } \\
\text { (Solid) }\end{array}$ & 96.77 & $\mathrm{~m} 3$ & 363.44 & $\mathrm{~m} 3$ & 460.21 & $\mathrm{~m} 3$ \\
\hline RCRA Regluated & 4.88 & $\mathrm{mt}$ & 94.73 & $\mathrm{mt}$ & 99.61 & $\mathrm{mt}$ \\
\hline State Regulated & 0.67 & $\mathbf{m t}$ & 13.02 & $\mathbf{m t}$ & 13.69 & $\mathrm{mt}$ \\
\hline TSCA Regulated & 0.00 & $\mathrm{mt}$ & 11.06 & $\mathrm{mt}$ & 11.06 & $\mathrm{mt}$ \\
\hline Mixed TSCA & 0.00 & $\mathrm{mt}$ & 4.67 & $\mathrm{mt}$ & 4.67 & $\mathrm{mt}$ \\
\hline
\end{tabular}




\section{Accomplishments Report}

Please Note: Only Accomplishments designated as FNAL will appear on this report !

Displaying sites: Hanford Site for calendar year 1999 for the Full Year

\section{Hanford Site}

\begin{tabular}{|c|c|c|c|}
\hline Waste Type & $\begin{array}{l}\text { Routine or } \\
\text { Cleanup/Stabilization }\end{array}$ & $\begin{array}{l}\text { Ongoing Project or } \\
\text { New Project }\end{array}$ & $\begin{array}{l}\text { Ongoing Project } \\
\text { Life (in years) }\end{array}$ \\
\hline Recycle/Reuse SAN & Routine & New & 0 \\
\hline
\end{tabular}

Waste Reduction

\begin{tabular}{|l|l|l|l|l|}
\hline Quarter 1 & Quarter 2 & Quarter 3 & Quarter 4 & YTD Reduction \\
\hline \hline $0.00 \mathrm{mt}$ & $0.00 \mathrm{mt}$ & $0.99 \mathrm{mt}$ & $0.00 \mathrm{mt}$ & $0.99 \mathrm{mt}$ \\
\hline & \multicolumn{1}{|c|}{ Savings } \\
\hline Quarter 1 & Quarter 2 & Quarter 3 & Quarter 4 & YTD Savings \\
\hline 0.00 & 0.00 & 131.00 & 0.00 & 131.00 \\
\hline
\end{tabular}

Accomplishment: Miscellaneous maintenance products that were redeployed through the Excess Chemical Program

\section{Hanford Site}

$\begin{array}{lllll}\text { P2 Activity } & \text { Waste Type } & \begin{array}{l}\text { Routine or } \\ \text { Cleanup/Stabilization }\end{array} & \begin{array}{l}\text { Ongoing Project or } \\ \text { New Project }\end{array} & \begin{array}{l}\text { Ongoing Project } \\ \text { Life (in years) }\end{array} \\ \begin{array}{llll}\text { Programmatic } \\ \text { Activities }\end{array} & \begin{array}{l}\text { Programmatic } \\ \text { Activities }\end{array} & \text { Routine } & \text { New } & 1\end{array}$

Waste Reduction

\begin{tabular}{|c|c|c|c|c|}
\hline Quarter 1 & Quarter 2 & Quarter 3 & Quarter 4 & YTD Reduction \\
\hline $0.00 \mathrm{~m} 3$ & $0.00 \mathrm{~m} 3$ & $0.00 \mathrm{~m} 3$ & $0.00 \mathrm{~m} 3$ & $0.00 \mathrm{~m} 3$ \\
\hline \multicolumn{5}{|c|}{ Savings } \\
\hline Quarter 1 & Quarter 2 & Quarter 3 & Quarter 4 & YTD Savings \\
\hline 0.00 & 0.00 & 0.00 & 0.00 & 0.00 \\
\hline
\end{tabular}

Accomplishment: Affirmative Procurement Training was developed for all buyers and Credit Card holders at Hanford. The training included discussion on (1) Definitions; (2) Application to Contractors; (3) Exclusions; (4) Regulations/Procedures; (5) Goals and Goal Progress; (6) Myths; (7) 
Case Studies; and (8) Resources. The training was delivered to over 100 buyers and 300 Credit Card holders. The training is ongoing as new Credit Card holders are assigned credit cards. At the request of Headquarters, the training was reformatted for other Department of Energy Sites to utilize.

\section{Hanford Site}

$\begin{array}{lllll}\text { P2 Activity } & \text { Waste Type } & \begin{array}{l}\text { Routine or } \\ \text { Cleanup/Stabilization }\end{array} & \begin{array}{l}\text { Ongoing Project or } \\ \text { New Project }\end{array} & \begin{array}{l}\text { Ongoing Project } \\ \text { Life (in years) }\end{array} \\ \begin{array}{l}\text { Programmatic } \\ \text { Activities }\end{array} & \begin{array}{l}\text { Programmatic } \\ \text { Activities }\end{array} & \text { Cleanup/Stabilization } & \text { New } & 1\end{array}$

Waste Reduction

\begin{tabular}{|l|l|l|l|l|}
\hline Quarter 1 & Quarter 2 & Quarter 3 & Quarter 4 & YTD Reduction \\
\hline $0.00 \mathrm{~m} 3$ & $0.00 \mathrm{~m} 3$ & $0.00 \mathrm{~m} 3$ & $0.00 \mathrm{~m} 3$ & $0.00 \mathrm{~m} 3$ \\
\hline
\end{tabular}

\section{Savings}

\begin{tabular}{|l|l|l|l|l|}
\hline Quarter 1 & Quarter 2 & Quarter 3 & Quarter 4 & YTD Savings \\
\hline \hline 0.00 & 0.00 & 0.00 & 0.00 & 0.00 \\
\hline
\end{tabular}

Accomplishment: An evaluation was performed to identify areas of environmental restoration activities that could lead to a potential volume reduction in support of the Environmental Restoration Project Waste Minimization/Pollution Prevention goals. The areas identified for potential reduction were analyzed through a Value Engineering approach which resulted in the development of certain pollution prevention opportunities, tailored to the Environmental Restoration Project. Construction equipment, concrete, structural steel, and soil waste streams were identified as the most viable for achieving waste minimization using the Value Engineering approach. Plans for implementation are being developed with the Department of Energy.

\section{Hanford Site}

\begin{tabular}{|c|c|c|c|c|}
\hline P2 Activity & Waste Type & $\begin{array}{l}\text { Routine or } \\
\text { Cleanup/Stabilization }\end{array}$ & $\begin{array}{l}\text { Ongoing Project or } \\
\text { New Project }\end{array}$ & $\begin{array}{l}\text { Ongoing Project } \\
\text { Life (in years) }\end{array}$ \\
\hline $\begin{array}{l}\text { Programmatic } \\
\text { Activities }\end{array}$ & $\begin{array}{l}\text { Programmatic } \\
\text { Activities }\end{array}$ & Cleanup/Stabilization & New & 1 \\
\hline
\end{tabular}

This accomplishment counts toward the DOE-wide reduction goal (i.e. 10\%) for waste resulting from cleanup, stabilization, and decommissioning activities.

Waste Reduction

\begin{tabular}{|c|c|c|c|c|}
\hline Quarter 1 & Quarter 2 & Quarter 3 & Quarter 4 & YTD Reduction \\
\hline $0.00 \mathrm{~m} 3$ & $0.00 \mathrm{~m} 3$ & $0.00 \mathrm{~m} 3$ & $0.00 \mathrm{~m} 3$ & $0.00 \mathrm{~m} 3$ \\
\hline
\end{tabular}




\begin{tabular}{|l|l|l|l|l|}
\hline Quarter 1 & Quarter 2 & Quarter 3 & Quarter 4 & YTD Savings \\
\hline \hline 0.00 & 0.00 & 0.00 & 0.00 & 0.00 \\
\hline
\end{tabular}

Accomplishment: An un-needed concrete crusher, attachment, and inventory of spare parts were transferred to the Mound Site in Ohio. The equipment will be used at several Department Of Energy sites at the Ohio Field Office for crushing concrete demolition waste from their environmental restoration activities. Basis: 77 metric tons replacement value $\$ 748,108$

\section{Hanford Site}

\begin{tabular}{|c|c|c|c|c|}
\hline P2 Activity & Waste Type & $\begin{array}{l}\text { Routine or } \\
\text { Cleanup/Stabilization }\end{array}$ & $\begin{array}{l}\text { Ongoing Project or } \\
\text { New Project }\end{array}$ & $\begin{array}{l}\text { Ongoing Project } \\
\text { Life (in years) }\end{array}$ \\
\hline $\begin{array}{l}\text { Programmatic } \\
\text { Activities }\end{array}$ & $\begin{array}{l}\text { Programmatic } \\
\text { Activities }\end{array}$ & Routine & New & 1 \\
\hline
\end{tabular}

Waste Reduction

\begin{tabular}{|l|l|l|l|l|}
\hline Quarter 1 & Quarter 2 & Quarter 3 & Quarter 4 & YTD Reduction \\
\hline $0.00 \mathrm{~m} 3$ & $0.00 \mathrm{~m} 3$ & $0.00 \mathrm{~m} 3$ & $0.00 \mathrm{~m} 3$ & $0.00 \mathrm{~m} 3$ \\
\hline
\end{tabular}

Savings

\begin{tabular}{|l|l|l|l|l|}
\hline Quarter 1 & Quarter 2 & Quarter 3 & Quarter 4 & YTD Savings \\
\hline \hline 0.00 & 0.00 & 0.00 & 0.00 & 0.00 \\
\hline
\end{tabular}

Accomplishment: As part of its Outreach activities, to promote public awareness in pollution prevention and waste minimization, the Waste Management Federal Services of Hanford, Inc. Pollution Prevention Group participates in the City of Richland's annual Earth Day celebration as well as the Hanford Site's annual Safety Exposition. Both events are held in the Spring. A Pollution Prevention Information Booth was maintained that offered a "Wheel of Pollution Prevention" game with prizes for the "correct" answer. The pollution prevention booth was quite popular with over 500 "players" estimated at the Earth Day event that had an estimated attendance of 6,000. Over 200 "players" were estimated at the Safety Exposition.

\section{Hanford Site}
P2 Activity
Waste Type Routine or
Ongoing Project or
Ongoing Project
Cleanup/Stabilization New Project
Life (in years)
Programmatic Programmatic
Activities Activities
Cleanup/Stabilization New 


\begin{tabular}{|l|l|l|l|l|}
\hline Quarter 1 & Quarter 2 & Quarter 3 & Quarter 4 & YTD Reduction \\
\hline $0.00 \mathrm{~m} 3$ & $0.00 \mathrm{~m} 3$ & $0.00 \mathrm{~m} 3$ & $0.00 \mathrm{~m} 3$ & $0.00 \mathrm{~m} 3$ \\
\hline \multicolumn{2}{|c|}{ Savings } \\
\hline
\end{tabular}

Accomplishment: At the request of Department of Energy Headquarters, Bechtel Hanford, Inc. supported a Pollution Prevention Program Assessment of the environmental restoration project at Rocky Flats Environmental Technology Site. The purpose of this review was two-fold 1) to support Department of Energy Headquarters during its annual review of Rocky Flats Environmental Technology Site Pollution Prevention Program and 2) to participate in a small waste minimization/cost savings pilot project assessment for three identified areas. The three areas studied were: removal and disposition of equipment in the Building 444, removal and disposition of depleted uranium stored in Building 444, and stabilization/recovery of transuranic and low level contaminated waste oils.

\section{Hanford Site}

\begin{tabular}{|c|c|c|c|c|}
\hline P2 Activity & Waste Type & $\begin{array}{l}\text { Routine or } \\
\text { Cleanup/Stabilization }\end{array}$ & $\begin{array}{l}\text { Ongoing Project or } \\
\text { New Project }\end{array}$ & $\begin{array}{l}\text { Ongoing Project } \\
\text { Life (in years) }\end{array}$ \\
\hline $\begin{array}{l}\text { Programmatic } \\
\text { Activities }\end{array}$ & $\begin{array}{l}\text { Programmatic } \\
\text { Actjvities. }\end{array}$ & Routine & New & 1 \\
\hline
\end{tabular}

Waste Reduction

\begin{tabular}{|c|c|c|c|c|}
\hline Quarter 1 & Quarter 2 & Quarter 3 & Quarter 4 & YTD Reduction \\
\hline $0.00 \mathrm{~m} 3$ & $0.00 \mathrm{~m} 3$ & $0.00 \mathrm{~m} 3$ & $0.00 \mathrm{~m} 3$ & $0.00 \mathrm{~m} 3$ \\
\hline \multicolumn{5}{|c|}{ Savings } \\
\hline Quarter 1 & Quarter 2 & Quarter 3 & Quarter 4 & YTD Savings \\
\hline 0.00 & 0.00 & 0.00 & 0.00 & 0.00 \\
\hline
\end{tabular}

Accomplishment: Fluor Hanford performed Pollution Prevention/Energy Efficiency (P2/E2) Training for Welch Foods, Inc., a fruit juice processing plant. The Kennewick plant processes approximately 6 million cases of frozen concentrate, juice, and spreads for distribution in the Western region. The Welch Foods, Inc. plant in Grandview, WA presses grapes $(\sim 100,000$ tons/year $)$ and apples $(\sim 40,000$ tons/year) sending the concentrates to plants in Kennewick, WA, Northeast, PA, and Lawton, MI for further processing. The training was specific to the Welch Foods, Inc. Kennewick and Grandview plants. Over 140 operators, technicians, managers, engineers, and administrative personnel attended the training. Included in the training was: (1) An introduction to Pollution Prevention; (2) The Environmental Protection Hierarchy of Pollution Prevention; (3) Techniques \& Examples; (4) An introduction to Energy Efficiency; (5) Barriers and Incentives to Pollution Prevention; and (6) Identification of Pollution Prevention/Energy Efficiency Solutions at Welch's. The success of the program was evaluated through the immense number of pollution prevention/energy efficiency opportunities identified in the training. Over 130 P2/E2 opportunities 
were put forth during the brainstorming session of the training. Many of the opportunities were simple to implement with little or no up front cost. All employees were required to attend the $1-1 / 2$ hour training session (10 sessions). The last half hour was dedicated to a facilitated brainstorming session to generate options for pollution prevention.

\section{Hanford Site}

\begin{tabular}{|c|c|c|c|c|}
\hline P2 Activity & Waste Type & $\begin{array}{l}\text { Routine or } \\
\text { Cleanup/Stabilization }\end{array}$ & $\begin{array}{l}\text { Ongoing Project or } \\
\text { New Project }\end{array}$ & $\begin{array}{l}\text { Ongoing Project } \\
\text { Life (in years) }\end{array}$ \\
\hline $\begin{array}{l}\text { Programmatic } \\
\text { Activities }\end{array}$ & $\begin{array}{l}\text { Programmatic } \\
\text { Activities }\end{array}$ & Routine & New & 1 \\
\hline
\end{tabular}

Waste Reduction

\begin{tabular}{|c|c|c|c|c|}
\hline Quarter 1 & Quarter 2 & Quarter 3 & Quarter 4 & YTD Reduction \\
\hline $0.00 \mathrm{~m} 3$ & $0.00 \mathrm{~m} 3$ & $0.00 \mathrm{~m} 3$ & $0.00 \mathrm{~m} 3$ & $0.00 \mathrm{~m} 3$ \\
\hline \multicolumn{5}{|c|}{ Savings } \\
\hline Quarter 1 & Quarter 2 & Quarter 3 & Quarter 4 & YTD Savings \\
\hline 0.00 & 0.00 & 0.00 & 0.00 & 0.00 \\
\hline
\end{tabular}

Accomplishment: Hanford's Pollution Prevention Small Business Program completed it's third successful year. Waste Management Hanford, worked with 10 small businesses in the cities of Kennewick and Richland. Among the industries were: food processing, concrete/asphalt manufacturing, hotel, golf course, apartment, floral, printing, and autobody. Of the 15 pollution prevention opportunities evaluated, 11 of the opportunities will be either implemented, or further evaluated by the business owner. Of the 15 pollution prevention opportunities evaluated for the City of Richland, 7 opportunities had a payback of less than 3 years. Total projected annual cost savings are $\$ 118,724$.

\section{Hanford Site}

\begin{tabular}{|c|c|c|c|c|}
\hline P2 Activity & Waste Type & $\begin{array}{l}\text { Routine or } \\
\text { Cleanup/Stabilization }\end{array}$ & $\begin{array}{l}\text { Ongoing Project or } \\
\text { New Project }\end{array}$ & $\begin{array}{l}\text { Ongoing Project } \\
\text { Life (in years) }\end{array}$ \\
\hline $\begin{array}{l}\text { Programmatic } \\
\text { Activities }\end{array}$ & $\begin{array}{l}\text { Programmatic } \\
\text { Activities }\end{array}$ & Routine & New & 1 \\
\hline
\end{tabular}

Waste Reduction

\begin{tabular}{|l|l|l|l|l|}
\hline Quarter 1 & Quarter 2 & Quarter 3 & Quarter 4 & YTD Reduction \\
\hline \hline $0.00 \mathrm{~m} 3$ & $0.00 \mathrm{~m} 3$ & $0.00 \mathrm{~m} 3$ & $0.00 \mathrm{~m} 3$ & $0.00 \mathrm{~m} 3$ \\
\hline
\end{tabular}




\begin{tabular}{|l|l|l|l|l|}
\hline \hline Quarter 1 & Quarter 2 & Quarter 3 & Quarter 4 & YTD Savings \\
\hline \hline 0.00 & 0.00 & 0.00 & 0.00 & 0.00 \\
\hline
\end{tabular}

Accomplishment: Major Hanford Site facilities, including K Basins, Tank Waste Remediation Systems, and Plutonium Finishing Plant, have incorporated the use of the Automated Job Hazard Analysis program. The program is a computerized pre-package review program. All hazards and anticipated waste are identified in advance, including Waste Minimization flags wherever possible. The programs application is designed to help work planners identify the possible hazards involved in a job task and also to implement the proper controls based on the hazards identified. This is accomplished by presenting the user with a panel of questions, which lead to controls based on the users responses. The program also provides the planner with a method of indicating participation of a Subject Matter Expert, by electronic signoffs. Waste/Min techniques are accomplished by indicating Waste Minimization controls for chemicals and/or waste being generated on the job through the Automated Job Hazard Analysis process.

\section{Hanford Site}

\begin{tabular}{|c|c|c|c|c|}
\hline P2 Activity & Waste Type & $\begin{array}{l}\text { Routine or } \\
\text { Cleanup/Stabilization }\end{array}$ & $\begin{array}{l}\text { Ongoing Project or } \\
\text { New Project }\end{array}$ & $\begin{array}{l}\text { Ongoing Project } \\
\text { Life (in years) }\end{array}$ \\
\hline $\begin{array}{l}\text { Programmatic } \\
\text { Activities }\end{array}$ & $\begin{array}{l}\text { Programmatic } \\
\text { Activities }\end{array}$ & Routine & New & 1 \\
\hline
\end{tabular}

Waste Reduction

\begin{tabular}{|c|c|c|c|c|}
\hline Quarter 1 & Quarter 2 & Quarter 3 & Quarter 4 & YTD Reduction \\
\hline $0.00 \mathrm{~m} 3$ & $0.00 \mathrm{~m} 3$ & $0.00 \mathrm{~m} 3$ & $0.00 \mathrm{~m} 3$ & $0.00 \mathrm{~m} 3$ \\
\hline \multicolumn{5}{|c|}{ Savings } \\
\hline Quarter 1 & Quarter 2 & $\overline{\text { Quarter 3 }}$ & Quarter 4 & YTD Savings \\
\hline 0.00 & 0.00 & 0.00 & $\sqrt{0.00}$ & $\sqrt{0.00}$ \\
\hline
\end{tabular}

Accomplishment: National Pollution Prevention Week (September 20 - 26, 1999) was held in conjunction with the City of Richland and local schools and businesses. An article was published in the Hanford Reach covering the week's events. A press release was sent to the local media promoting the week's events and activities. Home Depot co-sponsered the week's events and provided displays of environmentally friendly products. A "Kid's Day" and a coloring contest for the kids were also cosponsored. Household Hazardous Waste Collection was conducted by the City of Richland, and the public was invited to tour their local wastewater treatment plants. 


\section{Hanford Site}

\begin{tabular}{|c|c|c|c|c|}
\hline P2 Activity & Waste Type & $\begin{array}{l}\text { Routine or } \\
\text { Cleanup/Stabilization }\end{array}$ & $\begin{array}{l}\text { Ongoing Project or } \\
\text { New Project }\end{array}$ & $\begin{array}{l}\text { Ongoing Project } \\
\text { Life (in years) }\end{array}$ \\
\hline $\begin{array}{l}\text { Programmatic } \\
\text { Activities }\end{array}$ & $\begin{array}{l}\text { Programmatic } \\
\text { Activities }\end{array}$ & Routine & New & 1 \\
\hline
\end{tabular}

Waste Reduction

\begin{tabular}{|l|l|l|l||l|}
\hline Quarter 1 & Quarter 2 & Quarter 3 & Quarter 4 & YTD Reduction \\
\hline $0.00 \mathrm{~m} 3$ & $0.00 \mathrm{~m} 3$ & $0.00 \mathrm{~m} 3$ & $0.00 \mathrm{~m} 3$ & $0.00 \mathrm{m3}$ \\
\hline \multicolumn{3}{|c|}{ Savings } \\
\hline \hline Quarter 1 & Quarter 2 & Quarter 3 & Quarter 4 & YTD Savings \\
\hline 0.00 & 0.00 & 0.00 & 0.00 & 0.00 \\
\hline
\end{tabular}

Accomplishment: The Department of Energy, Richland Operations Office earned two of the highest awards for their leadership in pollution prevention activities at the Hanford Site. The Department of Energy has recognized Hanford's comprehensive public outreach program on pollution prevention as one of the best in the Department of Energy complex. This program sponsored several activities which informed people about pollution prevention and the impact of pollution on the environment. In addition, Department of Energy, Richland Operations Office was awarded a "White House Closing the Circle Award" for their efforts in developing a first of it's kind strategy to insure purchasing of products composed of recovered materials at the Hanford Site. The award recognizes efforts that set the example for other federal agencies and facilities to make significant contributions to a sustainable environment and reduce significant impacts on the environment.

\section{Hanford Site}
P2 Activity
Waste Type
Routine or
Ongoing Project or
Ongoing Project
Cleanup/Stabilization New Project
Life (in years)
Programmatic Programmatic
Activities
Activities
Cleanup/Stabilization New
1

Waste Reduction

\begin{tabular}{|l|l|l|l|l|}
\hline Quarter 1 & Quarter 2 & Quarter 3 & Quarter 4 & YTD Reduction \\
\hline $0.00 \mathrm{m3}$ & $0.00 \mathrm{~m} 3$ & $0.00 \mathrm{~m} 3$ & $0.00 \mathrm{~m} 3$ & $0.00 \mathrm{~m} 3$ \\
\hline \multicolumn{3}{|c|}{ Savings } \\
\hline \multicolumn{2}{|c|}{} & \multicolumn{2}{|c|}{} \\
\hline Quarter 1 & Quarter 2 & Quarter 3 & Quarter 4 & YTD Savings \\
\hline 0.00 & 0.00 & 0.00 & 0.00 & 0.00 \\
\hline
\end{tabular}

Accomplishment: The Environmental Restoration Contractor has incorporated site-specific waste management instructions in project documents. These documents provide guidance for the 
management of waste generated from the clean-out and demolition of the 105-F and DR Reactor Facilities. Also included are the identification of waste minimization opportunities and direction that the project perform recommended waste reducing techniques in order to reduce the amount of waste generated.

\section{Hanford Site}

$\begin{array}{lllll}\text { P2 Activity } & \text { Waste Type } & \begin{array}{l}\text { Routine or } \\ \text { Cleanup/Stabilization }\end{array} & \begin{array}{l}\text { Ongoing Project or } \\ \text { New Project }\end{array} & \begin{array}{l}\text { Ongoing Project } \\ \text { Life (in years) }\end{array} \\ \begin{array}{l}\text { Programmatic } \\ \text { Activities }\end{array} & \begin{array}{l}\text { Programmatic } \\ \text { Activities }\end{array} & \text { Routine } & \text { New } & 1\end{array}$

Waste Reduction

\begin{tabular}{|l|l|l|l|l||}
\hline Quarter 1 & Quarter 2 & Quarter 3 & Quarter 4 & YTD Reduction \\
\hline \hline $0.00 \mathrm{~m} 3$ & $0.00 \mathrm{~m} 3$ & $0.00 \mathrm{~m} 3$ & $0.00 \mathrm{~m} 3$ & $0.00 \mathrm{~m} 3$ \\
\hline
\end{tabular}

Savings

\begin{tabular}{|l|l|l|l|l|}
\hline Quarter 1 & Quarter 2 & Quarter 3 & Quarter 4 & YTD Savings \\
\hline \hline 0.00 & 0.00 & 0.00 & 0.00 & 0.00 \\
\hline
\end{tabular}

Accomplishment: The following site strategic Pollution Prevention Opportunity Assessments (PPOAs) were conducted during Calendar Year 1999: "Tank Waste Remediation Systems Diversion Pits MLLW/LLW", investigated the use of less waste intensive ventilation technology; "Waste Sampling and Characterization Facility (WSCF) MLLW/LLW Generation", examined various aspects of WSCF's sample acceptance and analytical procedures; "Fluorescent Light Ballast Containing Polychlorinated Biphenyls", investigated alternative lighting; "Analytical Waste Generation at 222S Laboratory", investigated less waste-intensive analytical technologies and techniques; "Alternative Machine Coolants", investigated the use of non-regulated coolants as well as air cooled machining technology; and "Tank Farms Operations and Maintenance Activities", investigated wood and plastic uses at Tank Farms. These PPOAs either had sitewide implications or were focused on reducing waste volumes for major generators. The opportunities identified as having a payback of three years or less have projected annual waste reductions of 9.6 cubic meters of LowLevel Waste, 2.7 cubic meters of Mixed Low-Level Waste, and 1.3 metric tons of hazardous waste. Total projected annual cost savings for these same opportunities are $\$ 199,000$.

\section{Hanford Site}

\begin{tabular}{|c|c|c|c|c|}
\hline P2 Activity & Waste Type & $\begin{array}{l}\text { Routine or } \\
\text { Cleanup/Stabilization }\end{array}$ & $\begin{array}{l}\text { Ongoing Project or } \\
\text { New Project }\end{array}$ & $\begin{array}{l}\text { Ongoing Project } \\
\text { Life (in years) }\end{array}$ \\
\hline $\begin{array}{l}\text { Programmatic } \\
\text { Activities }\end{array}$ & $\begin{array}{l}\text { Programmatic } \\
\text { Activities }\end{array}$ & Routine & New & 1 \\
\hline
\end{tabular}




\begin{tabular}{|l|l|l|l|l|}
\hline Quarter 1 & Quarter 2 & Quarter 3 & Quarter 4 & YTD Reduction \\
\hline $0.00 \mathrm{~m} 3$ & $0.00 \mathrm{~m} 3$ & $0.00 \mathrm{~m} 3$ & $0.00 \mathrm{~m} 3$ & $0.00 \mathrm{~m} 3$ \\
\hline
\end{tabular}

Savings

\begin{tabular}{|l|l|l|l|l|}
\hline Quarter 1 & Quarter 2 & Quarter 3 & Quarter 4 & YTD Savings \\
\hline 0.00 & 0.00 & 0.00 & 0.00 & 0.00 \\
\hline
\end{tabular}

Accomplishment: The Waste Management Federal Services of Hanford, Inc. Pollution Prevention Group and Department of Energy - Richland Operations Office (DOE-RL) assisted Oak Ridge National Laboratory in generating a report entitled, "Assessment of Cost Savings of DOE's Returnon-Investment Program", for Department of Energy Headquarters. The report focused on Oak Ridge National Laboratory's and Hanford Site's Return On Investment programs. Technical assistance was provided through a series of review and comment periods and interviews with project managers.

\section{Hanford Site}

$\begin{array}{lllll}\text { P2 Activity } & \text { Waste Type } & \begin{array}{l}\text { Routine or } \\ \text { Cleanup/Stabilization }\end{array} & \begin{array}{l}\text { Ongoing Project or } \\ \text { New Project }\end{array} & \begin{array}{l}\text { Ongoing Project } \\ \text { Life (in years) }\end{array} \\ \begin{array}{llll}\text { Programmatic Programmatic } & \text { Routine } & \text { New } & 1 \\ \text { Activities } & \text { Activities } & \text { Routine } & \end{array}\end{array}$

Waste Reduction

\begin{tabular}{|c|c|c|c|c|}
\hline Quarter 1 & Quarter 2 & Quarter 3 & Quarter 4 & YTD Reduction \\
\hline $0.00 \mathrm{~m} 3$ & $0.00 \mathrm{~m} 3$ & $0.00 \mathrm{~m} 3$ & $0.00 \mathrm{~m} 3$ & $0.00 \mathrm{~m} 3$ \\
\hline \multicolumn{5}{|c|}{ Savings } \\
\hline Quarter 1 & Quarter 2 & Quarter 3 & Quarter 4 & YTD Savings \\
\hline 0.00 & 0.00 & 0.00 & 0.00 & 0.00 \\
\hline
\end{tabular}

Accomplishment: Two FT Crowe Bridge Cranes (one 3 ton capacity and one 10 ton capacity) were removed from the 308 Building, and transferred to Tri-City Industrial Development Council (TRIDEC) for use in attracting new businesses to the local Tri-City area. Transfer was conducted through Economic Transition organization at Fluor Daniel Hanford Co.

\section{Hanford Site}

\begin{tabular}{|c|c|c|c|c|}
\hline P2 Activity & Waste Type & $\begin{array}{l}\text { Routine or } \\
\text { Cleanup/Stabilization }\end{array}$ & $\begin{array}{l}\text { Ongoing Project or } \\
\text { New Project }\end{array}$ & $\begin{array}{l}\text { Ongoing Project } \\
\text { Life (in years) }\end{array}$ \\
\hline $\begin{array}{l}\text { Programmatic } \\
\text { Activities }\end{array}$ & $\begin{array}{l}\text { Programmatic } \\
\text { Activities }\end{array}$ & Routine & New & 1 \\
\hline
\end{tabular}

Waste Reduction 


\begin{tabular}{|l|l|l|l|l|}
\hline Quarter 1 & Quarter 2 & Quarter 3 & Quarter 4 & YTD Reduction \\
\hline \hline $0.00 \mathrm{~m} 3$ & $0.00 \mathrm{~m} 3$ & $0.00 \mathrm{~m} 3$ & $0.00 \mathrm{~m} 3$ & $0.00 \mathrm{~m} 3$ \\
\hline \multicolumn{2}{|c|}{ Savings } \\
\hline \multicolumn{2}{|c|}{} & \multicolumn{2}{|c|}{} \\
\hline Quarter 1 & Quarter 2 & Quarter 3 & Quarter 4 & YTD Savings \\
\hline \hline 0.00 & 0.00 & 0.00 & 0.00 & 0.00 \\
\hline
\end{tabular}

Accomplishment: U.S. Department of Energy, Richland Operations Office (DOE-RL), with author, Waste Management Federal Services of Hanford,Inc. prepared and issued the Affirmative Procurement Strategy for the Hanford Site. This formal DOE-RL document includes complex- and site-wide practices, and analysis of Hanford's programs, and recommendations for meeting the 100percent goals. The strategy was posted on Hanford's internal webpage as well.

\section{Hanford Site}

$\begin{array}{lllll}\text { P2 Activity } & \text { Waste Type } & \begin{array}{l}\text { Routine or } \\ \text { Cleanup/Stabilization }\end{array} & \begin{array}{l}\text { Ongoing Project or } \\ \text { New Project }\end{array} & \begin{array}{l}\text { Ongoing Project } \\ \text { Life (in years) }\end{array} \\ \text { Recycle/Reuse HAZ } & \text { Cleanup/Stabilization } & \text { New } & 1\end{array}$

This accomplishment counts toward the DOE-wide reduction goal (i.e. 10\%) for waste resulting from cleanup, stabilization, and decommissioning activities.

Waste Reduction

\begin{tabular}{|c|c|c|c|c|}
\hline Quarter 1 & Quarter 2 & Quarter 3 & Quarter 4 & YTD Reduction \\
\hline $1.10 \mathrm{mt}$ & $0.00 \mathrm{mt}$ & $0.00 \mathrm{mt}$ & $0.00 \mathrm{mt}$ & $1.10 \mathrm{mt}$ \\
\hline \multicolumn{5}{|c|}{ Savings } \\
\hline Quarter 1 & Quarter 2 & Quarter 3 & Quarter 4 & YTD Savings \\
\hline $12,870.00$ & 0.00 & 0.00 & 0.00 & $12,870.00$ \\
\hline
\end{tabular}

Accomplishment: $105-\mathrm{C}$ recycled and N-Deactivation recycled used oil to an off site recycler.

\section{Hanford Site}

$\begin{array}{lllll}\text { P2 Activity } & \text { Waste Type } & \begin{array}{l}\text { Routine or } \\ \text { Cleanup/Stabilization }\end{array} & \begin{array}{l}\text { Ongoing Project or } \\ \text { New Project }\end{array} & \begin{array}{l}\text { Ongoing Project } \\ \text { Life (in years) }\end{array} \\ \text { Recycle/Reuse HAZ } & \text { Cleanup/Stabilization } & \text { New } & 1\end{array}$

This accomplishment counts toward the DOE-wide reduction goal (i.e. 10\%) for waste resulting from cleanup, stabilization, and decommissioning activities.

\section{Waste Reduction}




\begin{tabular}{|l|l|l|l|l|}
\hline Quarter 1 & Quarter 2 & Quarter 3 & Quarter 4 & YTD Reduction \\
\hline $0.00 \mathrm{mt}$ & $0.00 \mathrm{mt}$ & $0.00 \mathrm{mt}$ & $0.05 \mathrm{mt}$ & $0.05 \mathrm{mt}$ \\
\hline
\end{tabular}

Accomplishment: Laboratory standards and chemicals from the 3728 building were no longer needed and were re-distributed to other on site contractors (Waste Sampling and Characterization Facility and the Hammer Training Facility). A mixture of solid and liquid products made up this list of material transfered.

\section{Hanford Site}

$\begin{array}{lllll}\text { P2 Activity } & \text { Waste Type } & \begin{array}{l}\text { Routine or } \\ \text { Cleanup/Stabilization }\end{array} & \begin{array}{l}\text { Ongoing Project or } \\ \text { New Project }\end{array} & \begin{array}{l}\text { Ongoing Project } \\ \text { Life (in years) }\end{array} \\ \text { Recycle/Reuse HAZ } & \text { Routine } & \text { New } & 1\end{array}$

Waste Reduction

\begin{tabular}{|c|c|c|c|c|}
\hline Quarter 1 & Quarter 2 & Quarter 3 & Quarter 4 & YTD Reduction \\
\hline $0.00 \mathrm{mt}$ & $0.00 \mathrm{mt}$ & $0.00 \mathrm{mt}$ & $0.40 \mathrm{mt}$ & $0.40 \mathrm{mt}$ \\
\hline \multicolumn{5}{|c|}{ Savings } \\
\hline Quarter 1 & Quarter 2 & Quarter 3 & Quarter 4 & \begin{tabular}{|l|} 
YTD Savings \\
\end{tabular} \\
\hline 0.00 & 0.00 & 0.00 & $8,655.00$ & $8,655.00$ \\
\hline
\end{tabular}

Accomplishment: Liquid mercury and mercury switches shipped from the Centralized Consolidated Recycle Center to an offsite recycle vendor.

\section{Hanford Site}

$\begin{array}{lllll}\text { P2 Activity } & \text { Waste Type } & \begin{array}{l}\text { Routine or } \\ \text { Cleanup/Stabilization }\end{array} & \begin{array}{l}\text { Ongoing Project or } \\ \text { New Project }\end{array} & \begin{array}{l}\text { Ongoing Project } \\ \text { Life (in years) }\end{array} \\ \text { Recycle/Reuse HAZ } & \text { Cleanup/Stabilization New } & 1\end{array}$

This accomplishment counts toward the DOE-wide reduction goal (i.e. 10\%) for waste resulting from cleanup, stabilization, and decommissioning activities.

Waste Reduction

\begin{tabular}{|l|l|l|l|l|}
\hline Quarter 1 & Quarter 2 & Quarter 3 & Quarter 4 & YTD Reduction \\
\hline $0.00 \mathrm{mt}$ & $0.00 \mathrm{mt}$ & $0.01 \mathrm{mt}$ & $0.00 \mathrm{mt}$ & $0.01 \mathrm{mt}$ \\
\hline
\end{tabular}




\begin{tabular}{|l|l|l|l|l|}
\hline Quarter 1 & Quarter 2 & Quarter 3 & Quarter 4 & YTD Savings \\
\hline \hline 0.00 & 0.00 & 117.00 & 0.00 & 117.00 \\
\hline
\end{tabular}

Accomplishment: Lubricants from the 105-F Reactor Building were redistributed on-site for use by the "Mobile Mechanic".

\section{Hanford Site}

$\begin{array}{lllll}\text { P2 Activity } & \text { Waste Type } & \begin{array}{l}\text { Routine or } \\ \text { Cleanup/Stabilization }\end{array} & \begin{array}{l}\text { Ongoing Project or } \\ \text { New Project }\end{array} & \begin{array}{l}\text { Ongoing Project } \\ \text { Life (in years) }\end{array} \\ \text { Recycle/Reuse HAZ } & \text { Routine } & \text { New } & 1\end{array}$

Waste Reduction

\begin{tabular}{|c|c|c|c|c|}
\hline Quarter 1 & Quarter 2 & Quarter 3 & Quarter 4 & YTD Reduction \\
\hline $0.00 \mathrm{mt}$ & $0.00 \mathrm{mt}$ & $0.99 \mathrm{mt}$ & $0.34 \mathrm{mt}$ & $1.33 \mathrm{mt}$ \\
\hline \multicolumn{5}{|c|}{ Savings } \\
\hline Quarter 1 & Quarter 2 & Quarter 3 & Quarter 4 & YTD Savings \\
\hline 0.00 & 0.00 & $11,583.00$ & $7,303.00$ & $18,886.00$ \\
\hline
\end{tabular}

Accomplishment: Miscellaneous maintenance products that were redeployed through the Excess Chemical Program

\section{Hanford Site}

\begin{tabular}{lllll} 
P2 Activity & \multicolumn{1}{c}{ Waste Type } & $\begin{array}{l}\text { Routine or } \\
\text { Cleanup/Stabilization }\end{array}$ & $\begin{array}{l}\text { Ongoing Project or } \\
\text { New Project }\end{array}$ & $\begin{array}{l}\text { Ongoing Project } \\
\text { Life (in years) }\end{array}$ \\
Recycle/Reuse HAZ & Cleanup/Stabilization & New & 1
\end{tabular}

This accomplishment counts toward the DOE-wide reduction goal (i.e. 10\%) for waste resulting from cleanup, stabilization, and decommissioning activities.

Waste Reduction

\begin{tabular}{|c|c|c|c|c|}
\hline Quarter 1 & Quarter 2 & Quarter 3 & Quarter 4 & YTD Reduction \\
\hline $0.30 \mathrm{mt}$ & $0.00 \mathrm{mt}$ & $0.00 \mathrm{mt}$ & $0.00 \mathrm{mt}$ & $0.30 \mathrm{mt}$ \\
\hline \multicolumn{5}{|c|}{ Savings } \\
\hline Quarter 1 & Quarter 2 & Quarter 3 & Quarter 4 & YTD Savings \\
\hline $6,516.00$ & 0.00 & 0.00 & 0.00 & $6,616.00$ \\
\hline
\end{tabular}

Accomplishment: Nine air conditioners and heat pumps were removed from service at the 1706-N facilities and sent to the warehouse for future reuse rather than being sent for disposal. 


\section{Hanford Site}
P2 Activit
Waste Type
Routine or
Ongoing Project or
Ongoing Project
Recycle/Reuse HAZ
Cleanup/Stabilization New Project
Life (in years)
Cleanup/Stabilization New
1

This accomplishment counts toward the DOE-wide reduction goal (i.e. 10\%) for waste resulting from cleanup, stabilization, and decommissioning activities.

\begin{tabular}{|c|c|c|c|c|}
\hline & & & & \\
\hline Quarter 1 & Quarter 2 & Quarter 3 & Quarter 4 & YTD Reduction \\
\hline $0.00 \mathrm{mt}$ & $0.00 \mathrm{mt}$ & $0.00 \mathrm{mt}$ & $0.16 \mathrm{mt}$ & $0.16 \mathrm{mt}$ \\
\hline \multicolumn{5}{|c|}{ Savings } \\
\hline Quarter 1 & Quarter 2 & Quarter 3 & Quarter 4 & YTD Savings \\
\hline 0.00 & 0.00 & 0.00 & 173.00 & 173.00 \\
\hline
\end{tabular}

Accomplishment: Since the Chlorofluorocarbon-12 (CFC-12) refrigerant was removed from the chillers at the site, several maintenance evalutions, produce Hydrochlorofluorocarbon-134a (HFC134a) and oil wastes. (approximately 50 Gallons). This refrigerant/oil mixture was recycled rather than disposed of as hazardous waste.

\section{Hanford Site}

$\begin{array}{llll}\text { P2 Activity Waste Type } & \begin{array}{l}\text { Routine or } \\ \text { Cleanup/Stabilization }\end{array} & \begin{array}{l}\text { Ongoing Project or } \\ \text { New Project }\end{array} & \begin{array}{l}\text { Ongoing Project } \\ \text { Life (in years) }\end{array} \\ \text { Recycle/Reuse HAZ } & \text { Routine } & \text { New } & 1\end{array}$

Waste Reduction

\begin{tabular}{|c|c|c|c|c|}
\hline Quarter 1 & Quarter 2 & Quarter 3 & Quarter 4 & YTD Reduction \\
\hline $0.00 \mathrm{mt}$ & $0.00 \mathrm{mt}$ & $0.00 \mathrm{mt}$ & $0.12 \mathrm{mt}$ & $0.12 \mathrm{mt}$ \\
\hline \multicolumn{5}{|c|}{ Savings } \\
\hline Quarter 1 & Quarter 2 & Quarter 3 & Quarter 4 & YTD Savings \\
\hline 0.00 & 0.00 & 0.00 & $2,594.00$ & $2,594.00$ \\
\hline
\end{tabular}

Accomplishment: Spent fixer sent offsite for silver recovery. This recycling activity belongs to COGEMA Engineering Corporation. 


\section{Hanford Site}

$\begin{array}{lllll}\text { P2 Activity } & \text { Waste Type } & \begin{array}{l}\text { Routine or } \\ \text { Cleanup/Stabilization }\end{array} & \begin{array}{l}\text { Ongoing Project or } \\ \text { New Project }\end{array} & \begin{array}{l}\text { Ongoing Project } \\ \text { Life (in years) }\end{array} \\ \text { Recycle/Reuse HAZ } & \text { Cleanup/Stabilization } & \text { New } & 1\end{array}$

This accomplishment counts toward the DOE-wide reduction goal (i.e. 10\%) for waste resulting from cleanup, stabilization, and decommissioning activities.

Waste Reduction

\begin{tabular}{|l|l|l|l|l|}
\hline Quarter 1 & Quarter 2 & Quarter 3 & Quarter 4 & YTD Reduction \\
\hline $0.00 \mathrm{mt}$ & $0.00 \mathrm{mt}$ & $0.00 \mathrm{mt}$ & $1.20 \mathrm{mt}$ & $1.20 \mathrm{mt}$ \\
\hline
\end{tabular}

Savings

\begin{tabular}{|l|l|l|l|l|}
\hline Quarter 1 & Quarter 2 & Quarter 3 & Quarter 4 & YTD Savings \\
\hline 0.00 & 0.00 & 0.00 & $13,719.00$ & $13,719.00$ \\
\hline
\end{tabular}

Accomplishment: Transferred 215 gallons of Bartlett Polymeric Barrier System paint from the Plutonium Finishing Plant to Bechtel Hanford, Inc. The paint was identified as excess and was no longer needed. Savings based on paint weighing 12 pounds per gallon and a cost avoidance of $\$ 13,719$ for purchasing the same new paint.

\section{Hanford Site}

$\begin{array}{lllll}\text { P2 Activity } & \text { Waste Type } & \begin{array}{l}\text { Routine or } \\ \text { Cleanup/Stabilization }\end{array} & \begin{array}{l}\text { Ongoing Project or } \\ \text { New Project }\end{array} & \begin{array}{l}\text { Ongoing Project } \\ \text { Life (in years) }\end{array} \\ \text { Recycle/Reuse HAZ } & \text { Cleanup/Stabilization } & \text { New } & 1\end{array}$

This accomplishment counts toward the DOE-wide reduction goal (i.e. 10\%) for waste resulting from cleanup, stabilization, and decommissioning activities.

Waste Reduction

\begin{tabular}{|c|c|c|c|c|}
\hline Quarter 1 & Quarter 2 & Quarter 3 & Quarter 4 & YTD Reduction \\
\hline $0.00 \mathrm{mt}$ & $0.00 \mathrm{mt}$ & $13.70 \mathrm{mt}$ & $0.00 \mathrm{mt}$ & $13.70 \mathrm{mt}$ \\
\hline \multicolumn{5}{|c|}{ Savings } \\
\hline Quarter 1 & Quarter 2 & Quarter 3 & Quarter 4 & YTD Savings \\
\hline 0.00 & 0.00 & $160,000.00$ & 0.00 & $160,000.00$ \\
\hline
\end{tabular}

Accomplishment: Two carbon steel underground tanks were removed from the 300 Area. The diesel/water mixture resulting from cleaning the tanks was shipped to a recycler for recovery. 


\section{Hanford Site}

$\begin{array}{lllll}\text { P2 Activity } & \text { Waste Type } & \begin{array}{l}\text { Routine or } \\ \text { Cleanup/Stabilization }\end{array} & \begin{array}{l}\text { Ongoing Project or } \\ \text { New Project }\end{array} & \begin{array}{l}\text { Ongoing Project } \\ \text { Life (in years) }\end{array} \\ \text { Recycle/Reuse LLW } & \text { Routine } & \text { New } & 1\end{array}$

Waste Reduction

\begin{tabular}{|c|c|c|c|c|}
\hline Quarter 1 & Quarter 2 & Quarter 3 & Quarter 4 & YTD Reduction \\
\hline $0.00 \mathrm{~m} 3$ & $0.00 \mathrm{~m} 3$ & $0.20 \mathrm{~m} 3$ & $0.00 \mathrm{~m} 3$ & $0.20 \mathrm{~m} 3$ \\
\hline \multicolumn{5}{|c|}{ Savings } \\
\hline Quarter 1 & $\longdiv { \text { Quarter } 2 }$ & Quarter 3 & Quarter 4 & YTD Savings \\
\hline 0.00 & 0.00 & 390.00 & 0.00 & 390.00 \\
\hline
\end{tabular}

Accomplishment: 430 smoke detectors were returned to the vendor (Siemens Corp) to be recycled. Savings are based on cost for disposal as low level waste.

\section{Hanford Site}

$\begin{array}{lllll}\text { P2 Activity } & \text { Waste Type } & \text { Routine or } & \text { Ongoing Project or } & \text { Ongoing Project } \\ & - & \text { Cleanup/Stabilization } & \text { New Project } & \text { Life (in years) } \\ \text { Recycle/Reuse LLW } & \text { Routine } & \text { New } & 1\end{array}$

Waste Reduction

\begin{tabular}{|c|c|c|c|c|}
\hline Quarter 1 & Quarter 2 & Quarter 3 & Quarter 4 & YTD Reduction \\
\hline $0.00 \mathrm{~m} 3$ & $0.00 \mathrm{~m} 3$ & $0.00 \mathrm{~m} 3$ & $0.03 \mathrm{m3}$ & $0.03 \mathrm{~m} 3$ \\
\hline \multicolumn{5}{|c|}{ Savings } \\
\hline Quarter 1 & Quarter 2 & Quarter 3 & Quarter 4 & YTD Savings \\
\hline 0 & 0.00 & 0.00 & 140.00 & 140.00 \\
\hline
\end{tabular}

Accomplishment: 73 pounds of tritium powered Exit Signs were returned to manufacturer for recycle. Savings are based on cost for disposal as low level waste.

\section{Hanford Site}
P2 Activity Waste Type Routine or
Ongoing Project or
Ongoing Project
Cleanup/Stabilization New Project
Life (in years)
Recycle/Reuse LLW
Cleanup/Stabilization New
1 
This accomplishment counts toward the DOE-wide reduction goal (i.e. 10\%) for waste resulting from cleanup, stabilization, and decommissioning activities.

\begin{tabular}{|c|c|c|c|c|}
\hline Quarter 1 & Quarter 2 & Quarter 3 & Quarter 4 & YTD Reduction \\
\hline $0.00 \mathrm{~m} 3$ & $0.00 \mathrm{~m} 3$ & $0.05 \mathrm{m3}$ & $0.00 \mathrm{~m} 3$ & $0.05 \mathrm{~m} 3$ \\
\hline \multicolumn{5}{|c|}{ Savings } \\
\hline Quarter 1 & Quarter 2 & Quarter 3 & Quarter 4 & YTD Savings \\
\hline 0.00 & 0.00 & $1,300.00$ & 0.00 & $1,300.00$ \\
\hline
\end{tabular}

Accomplishment: The 222-S Facility Operations group had 13 gallons of a stripcoat which the facility no longer needed. The chemical was excessed to the As Low As Reasonably Achievable (ALARA) Center for redistribution throughout the Hanford Site:

\section{Hanford Site}

$\begin{array}{llll}\text { P2 Activity Waste Type } & \begin{array}{l}\text { Routine or } \\ \text { Cleanup/Stabilization }\end{array} & \begin{array}{l}\text { Ongoing Project or } \\ \text { New Project }\end{array} & \begin{array}{l}\text { Ongoing Project } \\ \text { Life (in years) }\end{array} \\ \text { Recycle/Reuse LLW } & \text { Cleanup/Stabilization } & \text { New } & 1\end{array}$

This accomplishment counts toward the DOE-wide reduction goal (i.e. 10\%) for waste resulting from cleanup, stabilization, and decommissioning activities.

Waste Reduction

\begin{tabular}{|c|c|c|c|c|}
\hline Quarter 1 & Quarter 2 & Quarter 3 & Quarter 4 & YTD Reduction \\
\hline $0.00 \mathrm{~m} 3$ & $0.00 \mathrm{~m} 3$ & $1.20 \mathrm{~m} 3$ & $0.00 \mathrm{~m} 3$ & $1.20 \mathrm{~m} 3$ \\
\hline \multicolumn{5}{|c|}{ Savings } \\
\hline Quarter 1 & Quarter 2 & Quarter 3 & Quarter 4 & \begin{tabular}{|l} 
YTD Savings \\
\end{tabular} \\
\hline 0.00 & 0.00 & $9,000.00$ & 0.00 & $9,000.00$ \\
\hline
\end{tabular}

Accomplishment: Two Z930 High-efficiency Particulate Air (HEPA) filter vacuums were excessed to the Hanford As Low as Reasonably Achievable (ALARA) Center. These vacuums are used for packaging large drums with waste.

\section{Hanford Site}

$\begin{array}{llll}\text { P2 Activity Waste Type } & \begin{array}{l}\text { Routine or } \\ \text { Cleanup/Stabilization }\end{array} & \begin{array}{l}\text { Ongoing Project or } \\ \text { New Project }\end{array} & \begin{array}{l}\text { Ongoing Project } \\ \text { Life (in years) }\end{array} \\ \text { Recycle/Reuse SAN } & \text { Routine } & \text { New } & 1\end{array}$

Waste Reduction 


\begin{tabular}{|l|l|l|l||l|}
\hline Quarter 1 & Quarter 2 & Quarter 3 & Quarter 4 & YTD Reduction \\
\hline $0.00 \mathrm{mt}$ & $0.20 \mathrm{mt}$ & $0.00 \mathrm{mt}$ & $0.00 \mathrm{mt}$ & $0.20 \mathrm{mt}$ \\
\hline & \multicolumn{2}{|c|}{ Savings } \\
\hline
\end{tabular}

Accomplishment: 10 Empty 30 gallon drums used to ship waste water to Liquid Effluent Facility for treatment are returned for reuse rather than sent for disposal

\section{Hanford Site}

$\begin{array}{lllll}\text { P2 Activity } & \text { Waste Type } & \begin{array}{l}\text { Routine or } \\ \text { Cleanup/Stabilization }\end{array} & \begin{array}{l}\text { Ongoing Project or } \\ \text { New Project }\end{array} & \begin{array}{l}\text { Ongoing Project } \\ \text { Life (in years) }\end{array} \\ \text { Recycle/Reuse SAN } & \text { Cleanup/Stabilization } & \text { New } & 1\end{array}$

This accomplishment counts toward the DOE-wide reduction goal (i.e. 10\%) for waste resulting from cleanup, stabilization, and decommissioning activities.

Waste Reduction

\begin{tabular}{|l|l|l|l|l|}
\hline Quarter 1 & Quarter 2 & Quarter 3 & Quarter 4 & YTD Reduction \\
\hline $5.10 \mathrm{mt}$ & $0.00 \mathrm{mt}$ & $0.00 \mathrm{mt}$ & $0.00 \mathrm{mt}$ & $5.10 \mathrm{mt}$ \\
\hline \multicolumn{3}{|c|}{ Savings } \\
\hline Quarter 1 & Quarter 2 & Quarter 3 & Quarter 4 & YTD Savings \\
\hline $7,250.00$ & 0.00 & 0.00 & 0.00 & $7,250.00$ \\
\hline
\end{tabular}

Accomplishment: 27 drums of antifreeze were shipped to Fire Systems Maintenance for reuse.

\section{Hanford Site}

$\begin{array}{llll}\text { P2 Activity } \quad \text { Waste Type } & \begin{array}{l}\text { Routine or } \\ \text { Cleanup/Stabilization }\end{array} & \begin{array}{l}\text { Ongoing Project or } \\ \text { New Project }\end{array} & \begin{array}{l}\text { Ongoing Project } \\ \text { Life (in years) }\end{array} \\ \text { Recycle/Reuse SAN } & \text { Cleanup/Stabilization } & \text { New } & 1\end{array}$

This accomplishment counts toward the DOE-wide reduction goal (i.e. 10\%) for waste resulting from cleanup, stabilization, and decommissioning activities.

Waste Reduction

\begin{tabular}{|l|l|l|l|l|}
\hline Quarter 1 & Quarter 2 & Quarter 3 & Quarter 4 & YTD Reduction \\
\hline $0.20 \mathrm{mt}$ & $0.00 \mathrm{mt}$ & $0.00 \mathrm{mt}$ & $0.00 \mathrm{mt}$ & $0.20 \mathrm{mt}$ \\
\hline
\end{tabular}




\begin{tabular}{|l|l||l|l||l||}
\hline Quarter 1 & Quarter 2 & Quarter 3 & Quarter 4 & YTD Savings \\
\hline \hline 126.00 & 0.00 & 0.00 & 0.00 & 126.00 \\
\hline \hline
\end{tabular}

Accomplishment: A large nylon reinforced plastic curtain draped in front of the front face of 105DR reactor to act as a heat deflector, was removed during decontamination and decommissioning activities. After a careful radiological survey, a portion was cut out and used as a safety banner which now hangs at the entrance to the project. The banner reads "Safety, because....". The remainder was used as a tarp to cover material.

\section{Hanford Site}

$\begin{array}{lllll}\text { P2 Activity } & \text { Waste Type } & \begin{array}{l}\text { Routine or } \\ \text { Cleanup/Stabilization }\end{array} & \begin{array}{l}\text { Ongoing Project or } \\ \text { New Project }\end{array} & \begin{array}{l}\text { Ongoing Project } \\ \text { Life (in years) }\end{array} \\ \text { Recycle/Reuse SAN } & \text { Cleanup/Stabilization } & \text { New } & 1\end{array}$

This accomplishment counts toward the DOE-wide reduction goal (i.e. 10\%) for waste resulting from cleanup, stabilization, and decommissioning activities.

Waste Reduction

\begin{tabular}{|c|c|c|c|c|}
\hline Quarter 1 & Quarter 2 & Quarter 3 & Quarter 4 & YTD Reduction \\
\hline $0.00 \mathrm{mt}$ & $0.00 \mathrm{mt}$ & $0.14 \mathrm{mt}$ & $0.00 \mathrm{mt}$ & $0.14 \mathrm{mt}$ \\
\hline \multicolumn{5}{|c|}{ Savings } \\
\hline Quarter 1 & Quarter 2 & Quarter 3 & Quarter 4 & YTD Savings \\
\hline 0.00 & 0.00 & 185.00 & 0.00 & 185.00 \\
\hline
\end{tabular}

Accomplishment: Floor cleaner that was previously declared as excess was utilized at different Environmental Restoration Contractor project sites.

\section{Hanford Site}

\begin{tabular}{|c|c|c|c|c|}
\hline P2 Activity & Waste Type & $\begin{array}{l}\text { Routine or } \\
\text { Cleanup/Stabilization }\end{array}$ & $\begin{array}{l}\text { Ongoing Project or } \\
\text { New Project }\end{array}$ & $\begin{array}{l}\text { Ongoing Project } \\
\text { Life (in years) }\end{array}$ \\
\hline & & Cleanup/Stabilization & New & 1 \\
\hline
\end{tabular}

This accomplishment counts toward the DOE-wide reduction goal (i.e. 10\%) for waste resulting from cleanup, stabilization, and decommissioning activities.

Waste Reduction

\begin{tabular}{|l|l|l|l||l|}
\hline Quarter 1 & Quarter 2 & Quarter 3 & Quarter 4 & YTD Reduction \\
\hline $0.00 \mathrm{mt}$ & $0.30 \mathrm{mt}$ & $0.00 \mathrm{mt}$ & $0.00 \mathrm{mt}$ & $0.30 \mathrm{mt}$ \\
\hline
\end{tabular}




\begin{tabular}{|l|l|l|l|l|}
\hline Quarter 1 & Quarter 2 & Quarter 3 & Quarter 4 & YTD Savings \\
\hline 0.00 & 243.00 & 0.00 & 0.00 & 243.00 \\
\hline
\end{tabular}

Accomplishment: Forty eight bags of Upright Safety Sorbent was sent off site to the Port of Ephrata (Federal Screener)

\section{Hanford Site}

$\begin{array}{lllll}\text { P2 Activity } & \text { Waste Type } & \begin{array}{l}\text { Routine or } \\ \text { Cleanup/Stabilization }\end{array} & \begin{array}{l}\text { Ongoing Project or } \\ \text { New Project }\end{array} & \begin{array}{l}\text { Ongoing Project } \\ \text { Life (in years) }\end{array} \\ \text { Recycle/Reuse SAN } & \text { Cleanup/Stabilization } & \text { New } & 1\end{array}$

This accomplishment counts toward the DOE-wide reduction goal (i.e. 10\%) for waste resulting from cleanup, stabilization, and decommissioning activities.

Waste Reduction

\begin{tabular}{|c|c|c|c|c|}
\hline Quarter 1 & Quarter 2 & Quarter 3 & Quarter 4 & YTD Reduction \\
\hline $0.00 \mathrm{mt}$ & $0.00 \mathrm{mt}$ & $0.00 \mathrm{mt}$ & $5.34 \mathrm{mt}$ & $5.34 \mathrm{mt}$ \\
\hline \multicolumn{5}{|c|}{ Savings } \\
\hline Quarter 1 & Quarter 2 & Quarter 3 & Quarter 4 & YTD Savings \\
\hline 0.00 & 0.00 & 0.00 & $4,454.00$ & $44,454.00$ \\
\hline
\end{tabular}

Accomplishment: Four compressed gas tanks measuring 24' diameter by 25 ' long were sent to an off site vendor for recycling.

\section{Hanford Site}

$\begin{array}{lllll}\text { P2 Activity } & \text { Waste Type } & \begin{array}{l}\text { Routine or } \\ \text { Cleanup/Stabilization }\end{array} & \begin{array}{l}\text { Ongoing Project or } \\ \text { New Project }\end{array} & \begin{array}{l}\text { Ongoing Project } \\ \text { Life (in years) }\end{array} \\ \text { Recycle/Reuse SAN } & \text { Cleanup/Stabilization New } & 1\end{array}$

This accomplishment counts toward the DOE-wide reduction goal (i.e. 10\%) for waste resulting from cleanup, stabilization, and decommissioning activities.

Waste Reduction

\begin{tabular}{|l|l|l|l|l|}
\hline Quarter 1 & Quarter 2 & Quarter 3 & Quarter 4 & YTD Reduction \\
\hline $1.70 \mathrm{mt}$ & $0.00 \mathrm{mt}$ & $0.00 \mathrm{mt}$ & $0.00 \mathrm{mt}$ & $1.70 \mathrm{mt}$ \\
\hline & \multicolumn{2}{|c|}{ Savings } \\
\hline \multicolumn{1}{|l|}{ Quarter 1 } & Quarter 2 & Quarter 3 & Quarter 4 & YTD Savings \\
\hline 124.00 & 0.00 & 0.00 & 0.00 & 124.00 \\
\hline
\end{tabular}


Accomplishment: Granular activated carbon was sent to offsite vendor for recycle.

\section{Hanford Site}

\begin{tabular}{|c|c|c|c|c|}
\hline P2 Activity & Waste Type & $\begin{array}{l}\text { Routine or } \\
\text { Cleanup/Stabilization }\end{array}$ & $\begin{array}{l}\text { Ongoing Project or } \\
\text { New Project }\end{array}$ & $\begin{array}{l}\text { Ongoing Project } \\
\text { Life (in years) }\end{array}$ \\
\hline Recycle/Reus & SAN & Cleanup/Stabilization & New & 1 \\
\hline
\end{tabular}

This accomplishment counts toward the DOE-wide reduction goal (i.e. 10\%) for waste resulting from cleanup, stabilization, and decommissioning activities.

Waste Reduction

\begin{tabular}{|c|c|c|c|c|}
\hline Quarter 1 & Quarter 2 & Quarter 3 & Quarter 4 & YTD Reduction \\
\hline $0.00 \mathrm{mt}$ & $0.00 \mathrm{mt}$ & $0.01 \mathrm{mt}$ & $0.00 \mathrm{mt}$ & $0.01 \mathrm{mt}$ \\
\hline \multicolumn{5}{|c|}{$\begin{array}{l}\text { Savings } \\
\end{array}$} \\
\hline Quarter 1 & Quarter 2 & Quarter 3 & Quarter 4 & YTD Savings \\
\hline 0.00 & 0.00 & 300.00 & 0.00 & 300.00 \\
\hline
\end{tabular}

Accomplishment: Graphic supplies that were previously declared as excess were utilized on site with other site contractors.

\section{Hanford Site}

\begin{tabular}{|c|c|c|c|c|}
\hline P2 Activity & Waste Type & $\begin{array}{l}\text { Routine or } \\
\text { Cleanup/Stabilization }\end{array}$ & $\begin{array}{l}\text { Ongoing Project or } \\
\text { New Project }\end{array}$ & $\begin{array}{l}\text { Ongoing Project } \\
\text { Life (in years) }\end{array}$ \\
\hline Recycle/Reu & SAN & Cleanup/Stabilization & New & 1 \\
\hline
\end{tabular}

This accomplishment counts toward the DOE-wide reduction goal (i.e. 10\%) for waste resulting from cleanup, stabilization, and decommissioning activities.

Waste Reduction

\begin{tabular}{|l|l|l|l|l|}
\hline Quarter 1 & Quarter 2 & Quarter 3 & Quarter 4 & YTD Reduction \\
\hline $9.00 \mathrm{mt}$ & $1.70 \mathrm{mt}$ & $0.00 \mathrm{mt}$ & $0.00 \mathrm{mt}$ & $10.70 \mathrm{mt}$ \\
\hline
\end{tabular}

Savings

\begin{tabular}{|l|l|l|l|l|}
\hline Quarter 1 & Quarter 2 & Quarter 3 & Quarter 4 & YTD Savings \\
\hline $1,788.00$ & 424.00 & 0.00 & 0.00 & $2,212.00$ \\
\hline
\end{tabular}

Accomplishment: Pallets and scrap wood were sent to an offsite contractor for recycle. 


\section{Hanford Site}
P2 Activity Waste Type Routine or
Ongoing Project or
Ongoing Project
Cleanup/Stabilization New Project
Recycle/Reuse SAN
Routine
New
Life (in years)
1

Waste Reduction

\begin{tabular}{|c|c|c|c|c|}
\hline Quarter 1 & Quarter 2 & Quarter 3 & Quarter 4 & YTD Reduction \\
\hline $0.05 \mathrm{mt}$ & $0.00 \mathrm{mt}$ & $0.00 \mathrm{mt}$ & $0.04 \mathrm{mt}$ & $0.09 \mathrm{mt}$ \\
\hline \multicolumn{5}{|c|}{ Savings } \\
\hline Quarter 1 & Quarter 2 & Quarter 3 & Quarter 4 & YTD Savings \\
\hline 7.00 & 0.00 & 0.00 & 6.00 & 13.00 \\
\hline
\end{tabular}

Accomplishment: Recycled viewgraphs through an offsite vendor.

\section{Hanford Site}

\begin{tabular}{|c|c|c|c|c|}
\hline P2 Activity & Waste Type & $\begin{array}{l}\text { Routine or } \\
\text { Cleanup/Stabilization }\end{array}$ & $\begin{array}{l}\text { Ongoing Project or } \\
\text { New Project }\end{array}$ & $\begin{array}{l}\text { Ongoing Project } \\
\text { Life (in years) }\end{array}$ \\
\hline Recycle/Reu & SAN & Cleanup/Stabilization & New & 1 \\
\hline
\end{tabular}

This accomplishment counts toward the DOE-wide reduction goal (i.e. 10\%) for waste resulting from cleanup, stabilization, and decommissioning activities.

Waste Reduction

\begin{tabular}{|c|c|c|c|c|}
\hline Quarter 1 & Quarter 2 & Quarter 3 & Quarter 4 & YTD Reduction \\
\hline $0.43 \mathrm{mt}$ & $0.00 \mathrm{mt}$ & $0.00 \mathrm{mt}$ & $0.00 \mathrm{mt}$ & $0.43 \mathrm{mt}$ \\
\hline \multicolumn{5}{|c|}{ Savings } \\
\hline Quarter 1 & Quarter 2 & Quarter 3 & Quarter 4 & YTD Savings \\
\hline 150.00 & 0.00 & 0.00 & 0.00 & 150.00 \\
\hline
\end{tabular}

Accomplishment: Sent propylene glycol to the Hammer Training Facility for use.

\section{Hanford Site}

$\begin{array}{llll}\text { P2 Activity Waste Type } & \begin{array}{l}\text { Routine or } \\ \text { Cleanup/Stabilization }\end{array} & \begin{array}{l}\text { Ongoing Project or } \\ \text { New Project }\end{array} & \begin{array}{l}\text { Ongoing Project } \\ \text { Life (in years) }\end{array} \\ \text { Recycle/Reuse SAN } & \text { Routine } & \text { New } & 1\end{array}$


Waste Reduction

\begin{tabular}{|l|l|l|l|l|}
\hline Quarter 1 & Quarter 2 & Quarter 3 & Quarter 4 & YTD Reduction \\
\hline $0.00 \mathrm{mt}$ & $0.00 \mathrm{mt}$ & $0.00 \mathrm{mt}$ & $12.01 \mathrm{mt}$ & $12.01 \mathrm{mt}$ \\
\hline \multicolumn{2}{|c|}{ Savings } \\
\hline
\end{tabular}

Accomplishment: Telephone books were recycled: 2.99 metric tons of US West given to Basin Recycling and 9.02 metric tons of GTE given to Richland School District

\section{Hanford Site}

$\begin{array}{lllll}\text { P2 Activity } & \text { Waste Type } & \begin{array}{l}\text { Routine or } \\ \text { Cleanup/Stabilization }\end{array} & \begin{array}{l}\text { Ongoing Project or } \\ \text { New Project }\end{array} & \begin{array}{l}\text { Ongoing Project } \\ \text { Life (in years) }\end{array} \\ \text { Recycle/Reuse SAN } & \text { Cleanup/Stabilization } & \text { New } & 1\end{array}$

This accomplishment counts toward the DOE-wide reduction goal (i.e. 10\%) for waste resulting from cleanup, stabilization, and decommissioning activities.

Waste Reduction

\begin{tabular}{|c|c|c|c|c|}
\hline Quarter 1 & Quarter 2 & Quarter 3 & Quarter 4 & YTD Reduction \\
\hline $0.00 \mathrm{mt}$ & $0.00 \mathrm{mt}$ & $0.00 \mathrm{mt}$ & $0.01 \mathrm{mt}$ & $0.01 \mathrm{mt}$ \\
\hline \multicolumn{5}{|c|}{ Savings } \\
\hline Quarter 1 & Quarter 2 & Quarter 3 & Quarter 4 & YTD Savings \\
\hline 0.00 & 0.00 & 0.00 & 83.00 & 83.00 \\
\hline
\end{tabular}

Accomplishment: Twenty five tubes of Zeniplex grease were obtained from another on-site contractor. The grease was identified as an excess material and was reclaimed for use on Bechtel Hanford, Inc. projects.

\section{Hanford Site}

\begin{tabular}{lllll} 
P2 Activity & \multicolumn{1}{c}{ Waste Type } & $\begin{array}{l}\text { Routine or } \\
\text { Cleanup/Stabilization }\end{array}$ & $\begin{array}{l}\text { Ongoing Project or } \\
\text { New Project }\end{array}$ & $\begin{array}{l}\text { Ongoing Project } \\
\text { Life (in years) }\end{array}$ \\
Recycle/Reuse SAN & Cleanup/Stabilization & New & 1
\end{tabular}

This accomplishment counts toward the DOE-wide reduction goal (i.e. 10\%) for waste resulting from cleanup, stabilization, and decommissioning activities. 


\begin{tabular}{|c|c|c|c|c|}
\hline Quarter 1 & Quarter 2 & Quarter 3 & Quarter 4 & YTD Reduction \\
\hline $0.00 \mathrm{mt}$ & $0.00 \mathrm{mt}$ & $21.77 \mathrm{mt}$ & $0.00 \mathrm{mt}$ & $21.77 \mathrm{mt}$ \\
\hline \multicolumn{5}{|c|}{ Savings } \\
\hline Quarter 1 & Quarter 2 & Quarter 3 & Quarter 4 & \begin{tabular}{|l|} 
YTD Savings \\
\end{tabular} \\
\hline 0.00 & 0.00 & $2,880.00$ & 0.00 & $2,880.00$ \\
\hline
\end{tabular}

Accomplishment: Two 16,000 gallon, carbon steel underground storage tanks were removed from the 300 Area and sent to Environmental Oil Co. to be recycled as scrap metal.

\section{Hanford Site}

$\begin{array}{lllll}\text { P2 Activity } & \text { Waste Type } & \begin{array}{l}\text { Routine or } \\ \text { Cleanup/Stabilization }\end{array} & \begin{array}{l}\text { Ongoing Project or } \\ \text { New Project }\end{array} & \begin{array}{l}\text { Ongoing Project } \\ \text { Life (in years) }\end{array} \\ \text { Recycle/Reuse WW-LLW } & \text { Cleanup/Stabilization } & \text { New } & 1\end{array}$

This accomplishment counts toward the DOE-wide reduction goal (i.e. 10\%) for waste resulting from cleanup, stabilization, and decommissioning activities.

Waste Reduction

\begin{tabular}{|c|c|c|c|c|}
\hline Quarter 1 & Quarter 2 & Quarter 3 & Quarter 4 & YTD Reduction \\
\hline $0.20 \mathrm{~m} 3$ & $0.00 \mathrm{m3}$ & $0.00 \mathrm{~m} 3$ & $0.00 \mathrm{~m} 3$ & $0.20 \mathrm{~m} 3$ \\
\hline \multicolumn{5}{|c|}{ Savings } \\
\hline Quarter 1 & Quarter 2 & Quarter 3 & Quarter 4 & YTD Savings \\
\hline 12.00 & 0.00 & 0.00 & 0.00 & 12.00 \\
\hline
\end{tabular}

Accomplishment: Marginally contaminated water from 233-S process systems was re-used in decontamination work rather than being sent to the Environmental Restoration Disposal Facility for disposal.

\section{Hanford Site}

$\begin{array}{lllll}\text { P2 Activity } & \text { Waste Type } & \begin{array}{l}\text { Routine or } \\ \text { Cleanup/Stabilization }\end{array} & \begin{array}{l}\text { Ongoing Project or } \\ \text { New Project }\end{array} & \begin{array}{l}\text { Ongoing Project } \\ \text { Life (in years) }\end{array} \\ \text { Segregation } & \text { LLW } & \text { Routine } & \text { New } & 1\end{array}$

Waste Reduction

\begin{tabular}{|l|l|l|l|l|}
\hline Quarter 1 & Quarter 2 & Quarter 3 & Quarter 4 & YTD Reduction \\
\hline $0.00 \mathrm{m3}$ & $0.00 \mathrm{m3}$ & $0.00 \mathrm{~m} 3$ & $815.00 \mathrm{~m} 3$ & $815.00 \mathrm{m3}$ \\
\hline
\end{tabular}




\begin{tabular}{|l|l|l|l|l|}
\hline Quarter 1 & Quarter 2 & Quarter 3 & Quarter 4 & YTD Savings \\
\hline 0.00 & 0.00 & 0.00 & $2,326,000.00$ & $2,326,000.00$ \\
\hline
\end{tabular}

Accomplishment: 58 metric tons of sanitary and 815 cubic meters of low-level waste disposal was avoided by the decontamination followed by the free-release and sale of 5 cranes by the DynCorp Crane and Rigging organization.

\section{Hanford Site}

$\begin{array}{lllll}\text { P2 Activity } & \text { Waste Type } & \begin{array}{l}\text { Routine or } \\ \text { Cleanup/Stabilization }\end{array} & \begin{array}{l}\text { Ongoing Project or } \\ \text { New Project }\end{array} & \begin{array}{l}\text { Ongoing Project } \\ \text { Life (in years) }\end{array} \\ \text { Segregation } & \text { LLW } & \text { Cleanup/Stabilization New } & 1 .\end{array}$

This accomplishment counts toward the DOE-wide reduction goal (i.e. 10\%) for waste resulting from cleanup, stabilization, and decommissioning activities.

\begin{tabular}{|c|c|c|c|c|}
\hline Quarter 1 & Quarter 2 & Quarter 3 & Quarter 4 & YTD Reduction \\
\hline $0.00 \mathrm{~m} 3$ & $0.00 \mathrm{~m} 3$ & $80.00 \mathrm{~m} 3$ & $0.00 \mathrm{~m} 3$ & $80.00 \mathrm{~m} 3$ \\
\hline \multicolumn{5}{|c|}{ Savings } \\
\hline Quarter 1 & Quarter 2 & Quarter 3 & Quarter 4 & YTD Savings \\
\hline 0.00 & 0.00 & $2,600.00$ & 0.00 & $2,600.00$ \\
\hline
\end{tabular}

Accomplishment: Concrete block rubble from the demolition of the 108-F Biological Laboratory was targeted to be disposed as Low Level Waste at the Environmental Restoration Disposal Facility. Approximately 80 cubic meters (166 metric ton) of potentially contaminated concrete block rubble generated from the demolition of the 108-F Biological Laboratory was re-used as clean backfill. Through a joint effort between Environmental Protection Agency, Department of Energy, Richland Operations Office, and Bechtel Hanford, Inc., the project was able to demonstrate that the rubble was free of contamination allowing it to be re-used. The rubble was transported to the $100 \mathrm{~B} / \mathrm{C}$ Remedial Action Site where it was used to fill in areas where contaminated soil was excavated during cleanup.

\section{Hanford Site}

$\begin{array}{lllll}\text { P2 Activity } & \text { Waste Type } & \begin{array}{l}\text { Routine or } \\ \text { Cleanup/Stabilization }\end{array} & \begin{array}{l}\text { Ongoing Project or } \\ \text { New Project }\end{array} & \begin{array}{l}\text { Ongoing Project } \\ \text { Life (in years) }\end{array} \\ \text { Segregation } & \text { LLW } & \text { Routine } & \text { New } & 1\end{array}$

Waste Reduction

\begin{tabular}{|l|l|l|l|l|}
\hline \hline Quarter 1 & Quarter 2 & Quarter 3 & Quarter 4 & YTD Reduction \\
\hline $37.65 \mathrm{~m} 3$ & $0.00 \mathrm{~m} 3$ & $0.00 \mathrm{~m} 3$ & $0.00 \mathrm{~m} 3$ & $37.65 \mathrm{~m} 3$ \\
\hline
\end{tabular}


Savings

\begin{tabular}{|l|l|l|l|l|}
\hline Quarter 1 & Quarter 2 & Quarter 3 & Quarter 4 & YTD Savings \\
\hline \hline $75,300.00$ & 0.00 & 0.00 & 0.00 & $75,300.00$ \\
\hline
\end{tabular}

Accomplishment: Crane \#17T-19959 was free released and therefore is no longer considered radiologically contaminated equipment. Crane was steam cleaned at $2711 \mathrm{E}$ and will be sold through the Materials Management organization.

\section{Hanford Site}

$\begin{array}{lllll}\text { P2 Activity } & \text { Waste Type } & \begin{array}{l}\text { Routine or } \\ \text { Cleanup/Stabilization }\end{array} & \begin{array}{l}\text { Ongoing Project or } \\ \text { New Project }\end{array} & \begin{array}{l}\text { Ongoing Project } \\ \text { Life (in years) }\end{array} \\ \text { Segregation } & \text { LLW } & \text { Cleanup/Stabilization } & \text { New } & 1\end{array}$

This accomplishment counts toward the DOE-wide reduction goal (i.e. 10\%) for waste resulting from cleanup, stabilization, and decommissioning activities.

Waste Reduction

\begin{tabular}{|c|c|c|c|c|}
\hline Quarter 1 & Quarter 2 & Quarter 3 & Quarter 4 & YTD Reduction \\
\hline $0.00 \mathrm{~m} 3$ & $0.00 \mathrm{~m} 3$ & $10.00 \mathrm{~m} 3$ & $0.00 \mathrm{~m} 3$ & $10.00 \mathrm{~m} 3$ \\
\hline \multicolumn{5}{|c|}{ Savings } \\
\hline Quarter 1 & Quarter 2 & Quarter 3 & Quarter 4 & YTD Savings \\
\hline 0.00 & 0.00 & $20,000.00$ & 0.00 & $20,000.00$ \\
\hline
\end{tabular}

Accomplishment: The compressor (W97D37873) was free released and excessed.

\section{Hanford Site}

$\begin{array}{lllll}\text { P2 Activity } & \text { Waste Type } & \begin{array}{l}\text { Routine or } \\ \text { Cleanup/Stabilization }\end{array} & \begin{array}{l}\text { Ongoing Project or } \\ \text { New Project }\end{array} & \begin{array}{l}\text { Ongoing Project } \\ \text { Life (in years) }\end{array} \\ \text { Segregation } & \text { LLW } & \text { Cleanup/Stabilization } & \text { New } & 1\end{array}$

Waste Reduction

\begin{tabular}{|c|c|c|c|c|}
\hline Quarter 1 & Quarter 2 & Quarter 3 & Quarter 4 & YTD Reduction \\
\hline $0.00 \mathrm{~m} 3$ & $0.00 \mathrm{~m} 3$ & $71,200.00 \mathrm{~m} 3$ & $0.00 \mathrm{~m} 3$ & $71,200.00 \mathrm{~m} 3$ \\
\hline \multicolumn{5}{|c|}{ Savings } \\
\hline Quarter 1 & Quarter 2 & Quarter 3 & Quarter 4 & YTD Savings \\
\hline 0.00 & 0.00 & $5,118,246.00$ & 0.00 & $5,118,246.00$ \\
\hline
\end{tabular}

Accomplishment: Utilizing a GR-130 Gamma Spectrometer and a E-600 survey instrument 
developed with Return on Investment project funding, the excavation of contaminated soil was minimized at the 100-D Area Group 2 Pipeline Project. The identification and extent of a contamination plume in the soil from pipelines known to be contaminated was not known prior to project startup. The successful implementation of these new, innovative instruments better identified the spread of contamination and minimized the amount of soil needing remediation. As a result of this effort, 93 percent of the soil excavated was determined to be free of contamination and used as clean backfill.

\section{Hanford Site}

$\begin{array}{lllll}\text { P2 Activity } & \text { Waste Type } & \begin{array}{l}\text { Routine or } \\ \text { Cleanup/Stabilization }\end{array} & \begin{array}{l}\text { Ongoing Project or } \\ \text { New Project }\end{array} & \begin{array}{l}\text { Ongoing Project } \\ \text { Life (in years) }\end{array}\end{array}$

Source

Reduction

HAZ

Cleanup/Stabilization New

1

This accomplishment counts toward the DOE-wide reduction goal (i.e. 10\%) for waste resulting from cleanup, stabilization, and decommissioning activities.

Waste Reduction

\begin{tabular}{|l|l|l|l|l|}
\hline Quarter 1 & Quarter 2 & Quarter 3 & Quarter 4 & YTD Reduction \\
\hline \hline $0.00 \mathrm{mt}$ & $0.00 \mathrm{mt}$ & $0.01 \mathrm{mt}$ & $0.00 \mathrm{mt}$ & $0.01 \mathrm{mt}$ \\
\hline
\end{tabular}

Savings

\begin{tabular}{|l|l|l|l|l|}
\hline Quarter 1 & Quarter 2 & Quarter 3 & Quarter 4 & YTD Savings \\
\hline 0.00 & 0.00 & 117.00 & 0.00 & 117.00 \\
\hline
\end{tabular}

Accomplishment: A new ice maker was purchased to replace one that broke. One of the factors that was considered when purchasing the new machine was the type of refrigerant used. It was decided that a machine containing an environmental friendly refrigerant (GENETRON 404A(R)) would be the ice maker purchased.

\section{Hanford Site}

$\begin{array}{lllll}\text { P2 Activity } & \text { Waste Type } & \begin{array}{l}\text { Routine or } \\ \text { Cleanup/Stabilization }\end{array} & \begin{array}{l}\text { Ongoing Project or } \\ \text { New Project }\end{array} & \begin{array}{l}\text { Ongoing Project } \\ \text { Life (in years) }\end{array} \\ \begin{array}{l}\text { Source } \\ \text { Reduction }\end{array} & \text { LLW } & \text { Cleanup/Stabilization } & \text { New } & 1\end{array}$

This accomplishment counts toward the DOE-wide reduction goal (i.e. 10\%) for waste resulting from cleanup, stabilization, and decommissioning activities.

Waste Reduction

\begin{tabular}{|l|l|l|l|l|}
\hline Quarter 1 & Quarter 2 & Quarter 3 & Quarter 4 & YTD Reduction \\
\hline \hline $0.00 \mathrm{~m} 3$ & $0.00 \mathrm{~m} 3$ & $29.40 \mathrm{~m} 3$ & $0.00 \mathrm{~m} 3$ & $29.40 \mathrm{~m} 3$ \\
\hline
\end{tabular}


Savings

\begin{tabular}{|l|l|l|l|l|}
\hline Quarter 1 & Quarter 2 & Quarter 3 & Quarter 4 & YTD Savings \\
\hline 0.00 & 0.00 & $131,652.00$ & 0.00 & $131,652.00$ \\
\hline
\end{tabular}

Accomplishment: 143,193 square feet of contamination area adjacent to diversion box 241-ER-151 were surveyed and released. The contaminated vegetation formerly collected from this area and disposed to burial was eliminated.

\section{Hanford Site}

$\begin{array}{lllll}\text { P2 Activity } & \text { Waste Type } & \begin{array}{l}\text { Routine or } \\ \text { Cleanup/Stabilization }\end{array} & \begin{array}{l}\text { Ongoing Project or } \\ \text { New Project }\end{array} & \begin{array}{l}\text { Ongoing Project } \\ \text { Life (in years) }\end{array} \\ \begin{array}{l}\text { Source } \\ \text { Reduction }\end{array} & \text { LLW } & \text { Routine } & \text { New } & 1\end{array}$

Waste Reduction

\begin{tabular}{|l|l|l|l|l|}
\hline \hline Quarter 1 & Quarter 2 & Quarter 3 & Quarter 4 & YTD Reduction \\
\hline $0.00 \mathrm{~m} 3$ & $0.00 \mathrm{~m} 3$ & $84.90 \mathrm{~m} 3$ & $0.00 \mathrm{~m} 3$ & $84.90 \mathrm{~m} 3$ \\
\hline
\end{tabular}

Savings

\begin{tabular}{|l|l|l|l|l|}
\hline \hline Quarter 1 & Quarter 2 & Quarter 3 & Quarter 4 & YTD Savings \\
\hline 0.00 & 0.00 & $161,310.00$ & 0.00 & $161,310.00$ \\
\hline
\end{tabular}

Accomplishment: Category 3 Low-Level Waste (LLW) was stabilized using flood grouting in the trench as an alternative to packaging the waste in high integrity containers (HICs). The activity was funded as a Return On Investment project. 43.5 cubic meters of Category 3 LLW was stabilized in an encasement making a total disposal volume of 69.6 cubic meters. The HIC stabilization method would have resulted in a disposal volume of 154.5 cubic meters for a waste volume reduction of 84.9 cubic meters.

\section{Hanford Site}

$\begin{array}{lllll}\text { P2 Activity } & \text { Waste Type } & \begin{array}{l}\text { Routine or } \\ \text { Cleanup/Stabilization }\end{array} & \begin{array}{l}\text { Ongoing Project or } \\ \text { New Project }\end{array} & \begin{array}{l}\text { Ongoing Project } \\ \text { Life (in years) }\end{array} \\ \begin{array}{l}\text { Source } \\ \text { Reduction }\end{array} & \text { LLW } & \text { Cleanup/Stabilization } & \text { New } & 1\end{array}$

Waste Reduction

\begin{tabular}{|l|l|l|l|l||}
\hline Quarter 1 & Quarter 2 & Quarter 3 & Quarter 4 & YTD Reduction \\
\hline $0.00 \mathrm{~m} 3$ & $0.00 \mathrm{~m} 3$ & $48,624.00 \mathrm{m3}$ & $0.00 \mathrm{~m} 3$ & $48,624.00 \mathrm{~m} 3$ \\
\hline
\end{tabular}




\begin{tabular}{|l|l|l|l|l|}
\hline \hline Quarter 1 & Quarter 2 & Quarter 3 & Quarter 4 & YTD Savings \\
\hline \hline 0.00 & 0.00 & $36,300,000.00$ & 0.00 & $36,300,000.00$ \\
\hline
\end{tabular}

Accomplishment: In the Fall of 1998, an effort was initiated to evaluate the waste sites identified in the 300-FF-2 Operable Unit following the waste site reclassification process, as described in the TriParty Agreement Handbook, Management Procedure TPA-MP-14, "Maintenance of the Waste Information Data System." The disposition of 417 waste sites in the 300-FF-2 Operable Unit was completed at the end of February 1999, following the waste categorization process. The process resulted in a substantial percentage of the waste sites requiring no further action. Because many of the sites were previously targeted for remedial action in 1999 Environmental Restoration Project baseline report, the disposition process resulted in a considerable cost avoidance. For the sites that have been estimated and are identified in the 1999 Environmental Restoration Project baseline (129 sites), an estimated cost avoidance of $\$ 15.3$ million resulted from the categorization process. For the 288 sites not identified in the 1999 Environmental Restoration Project baseline, an avoidance of \$21.2 million is estimated. The basis for arriving at this value is a comparison to other analogous sites that had previously been estimated.

\section{Hanford Site}

$\begin{array}{lllll}\text { P2 Activity } & \text { Waste Type } & \begin{array}{l}\text { Routine or } \\ \text { Cleanup/Stabilization }\end{array} & \begin{array}{l}\text { Ongoing Project or } \\ \text { New Project }\end{array} & \begin{array}{l}\text { Ongoing Project } \\ \text { Life (in years) }\end{array} \\ \begin{array}{l}\text { Source } \\ \text { Reduction }\end{array} & \text { LLW } & \text { Routine } & \text { New } & 1\end{array}$

Waste Reduction

\begin{tabular}{|c|c|c|c|c|}
\hline Quarter 1 & Quarter 2 & Quarter 3 & Quarter 4 & YTD Reduction \\
\hline $0.00 \mathrm{~m} 3$ & $0.01 \mathrm{~m} 3$ & $0.00 \mathrm{~m} 3$ & $35.80 \mathrm{~m} 3$ & $35.81 \mathrm{~m} 3$ \\
\hline \multicolumn{5}{|c|}{ Savings } \\
\hline Quarter 1 & Quarter 2 & Quarter 3 & Quarter 4 & YTD Savings \\
\hline 0.00 & 200.00 & 0.00 & $157,982.00$ & $158,182.00$ \\
\hline
\end{tabular}

Accomplishment: Surveyed, cleaned and down posted 1000 square feet at 152-ER and 36 square feet at 216-A-40 from a radiological controlled area to a radiological buffer area. Reduced waste from personal protective equipment and maintenance activities. During the Fourth Quarter an additional $35.8 \mathrm{~m} 3$ were downposted.

\section{Hanford Site}
P2 Activity
Waste Type Routine or
Ongoing Project or
Ongoing Project 
Source

Reduction

LLW

Cleanup/Stabilization New

1

Waste Reduction

\begin{tabular}{|c|c|c|c|c|}
\hline Quarter 1 & Quarter 2 & Quarter 3 & Quarter 4 & YTD Reduction \\
\hline $7,000.00 \mathrm{~m} 3$ & $0.00 \mathrm{~m} 3$ & $0.00 \mathrm{~m} 3$ & $0.00 \mathrm{~m} 3$ & $7,000.00 \mathrm{~m} 3$ \\
\hline \multicolumn{5}{|c|}{ Savings } \\
\hline Quarter 1 & Quarter 2 & Quarter 3 & Quarter 4 & YTD Savings \\
\hline $628,215.00$ & 0.00 & 0.00 & 0.00 & $628,215.00$ \\
\hline
\end{tabular}

Accomplishment: The original group 3 remedial action design specified a 1-meter-thick layer of potentially clean overburden at the 116-B-4 plume areas, 116-B-2, 116-B-12, and the 116-C-2A, B, and $C$ sites. The approximate original design volume of potentially clean overburden was 4,700 cubic meters. The Environmental Restoration Contractor analytical team was able to use return on investment funded field screening technologies (Eberline 600 Radiological Survey Meters and NaI, Exploranium GP-130 Handheld Gamma Ray Spectrometers) and "pothole" excavations to better define the thickness of the clean overburden. The volume of overburden identified as potentially clean was increased by a factor of approximately 2.5 . The approximate actual volume of soils set aside as potentially clean overburden is 11,700 cubic meters; an increase of over 7,000 cubic meters. Finding an additional 7000 cubic meters of clean overburden means 7000 cubic meters of soil that does not need to be shipped to the Environmental Restoration Disposal Facility.

\section{Hanford Site}

$\begin{array}{lllll}\text { P2 Activity } & \text { Waste Type } & \begin{array}{l}\text { Routine or } \\ \text { Cleanup/Stabilization }\end{array} & \begin{array}{l}\text { Ongoing Project or } \\ \text { New Project }\end{array} & \begin{array}{l}\text { Ongoing Project } \\ \text { Life (in years) }\end{array} \\ \begin{array}{l}\text { Source. } \\ \text { Reduction }\end{array} & \text { MLLW } & \text {. Routine } & \text { New } & 1\end{array}$

Waste Reduction

\begin{tabular}{|l|l|l|l|l|}
\hline Quarter 1 & Quarter 2 & Quarter 3 & Quarter 4 & YTD Reduction \\
\hline $0.00 \mathrm{m3}$ & $0.00 \mathrm{m3}$ & $0.01 \mathrm{~m} 3$ & $0.00 \mathrm{~m} 3$ & $0.01 \mathrm{m3}$ \\
\hline
\end{tabular}

Savings

\begin{tabular}{|l|l|l|l|l|}
\hline Quarter 1 & Quarter 2 & Quarter 3 & Quarter 4 & YTD Savings \\
\hline 0.00 & 0.00 & $25,993.00$ & 0.00 & $25,993.00$ \\
\hline
\end{tabular}

Accomplishment: A solid-state ammonia electrode was purchased to evaluate a new method for ammonia measurement. The new method consists of measuring ammonia in the off-gas rather than the liquid, resulting in a substantial reduction in probes required to perform analyses on Tank Farm samples. 


\section{Hanford Site}

$\begin{array}{lllll}\text { P2 Activity } & \text { Waste Type } & \begin{array}{l}\text { Routine or } \\ \text { Cleanup/Stabilization }\end{array} & \begin{array}{l}\text { Ongoing Project or } \\ \text { New Project }\end{array} & \begin{array}{l}\text { Ongoing Project } \\ \text { Life (in years) }\end{array} \\ \begin{array}{l}\text { Source } \\ \text { Reduction }\end{array} & \text { MLLW } & \text { Routine } & \text { New } & 1\end{array}$

Waste Reduction

\begin{tabular}{|l|l|l|l|l|}
\hline Quarter 1 & Quarter 2 & Quarter 3 & Quarter 4 & YTD Reduction \\
\hline \hline $0.00 \mathrm{~m} 3$ & $0.00 \mathrm{~m} 3$ & $0.10 \mathrm{~m} 3$ & $0.10 \mathrm{~m} 3$ & $0.20 \mathrm{~m} 3$ \\
\hline
\end{tabular}

Savings

\begin{tabular}{|l|l|l|l|l|}
\hline Quarter 1 & Quarter 2 & Quarter 3 & Quarter 4 & YTD Savings \\
\hline \hline 0.00 & 0.00 & 300.00 & 700.00 & $1,000.00$ \\
\hline
\end{tabular}

Accomplishment: Waste Receiving and Processing (WRAP) Environmental Compliance periodically reviews maintenance and operations activities associated with the process area (Buffer Zone \& Control Area (CA)) chemical use. As a result, WRAP has been succesful at preventing facility mixed waste generation by providing acceptable work controls which allow for the free release of chemicals.

\section{Hanford Site}

$\begin{array}{lllll}\text { P2 Activity } & \text { Waste Type } & \begin{array}{l}\text { Routine or } \\ \text { Cleanup/Stabilization }\end{array} & \begin{array}{l}\text { Ongoing Project or } \\ \text { New Project }\end{array} & \begin{array}{l}\text { Ongoing Project } \\ \text { Life (in years) }\end{array} \\ \begin{array}{l}\text { Source } \\ \text { Reduction }\end{array} & \text { WW-LLW } & \text { Cleanup/Stabilization } & \text { New } & 1\end{array}$

This accomplishment counts toward the DOE-wide reduction goal (i.e. 10\%) for waste resulting from cleanup, stabilization, and decommissioning activities.

Waste Reduction

\begin{tabular}{|l|l||l|l||l|}
\hline Quarter 1 & Quarter 2 & Quarter 3 & Quarter 4 & YTD Reduction \\
\hline $0.00 \mathrm{~m} 3$ & $53.14 \mathrm{m3}$ & $0.00 \mathrm{~m} 3$ & $0.00 \mathrm{~m} 3$ & $53.14 \mathrm{~m} 3$ \\
\hline
\end{tabular}

Savings

\begin{tabular}{|l|l|l|l|l|}
\hline Quarter 1 & Quarter 2 & Quarter 3 & Quarter 4 & YTD Savings \\
\hline \hline 0.00 & $3,509.00$ & 0.00 & 0.00 & $3,509.00$ \\
\hline
\end{tabular}

Accomplishment: The 1300-N Emergency Dump Basin is an outdoor liquid effluent storage basin that was originally designed to receive emergency cooling water from the $\mathrm{N}$ Reactor. The basin is approximately 40 meters ( 130 feet) long by 24 meters ( 80 feet) wide by 9 meters ( 30 feet) deep at the center, and has a storage capacity of about 3.8 million liters ( 1 million gallons). The basin has a carbon-steel liner. During the deactivation of the Emergency Dump Basin, a geomembrane liner was 
installed to isolate the contaminated surface of the basin from potential rain water that would eventually collect in the basin and become contaminated.

\section{Hanford Site}

$\begin{array}{lllll}\text { P2 Activity } & \text { Waste Type } & \begin{array}{l}\text { Routine or } \\ \text { Cleanup/Stabilization }\end{array} & \begin{array}{l}\text { Ongoing Project or } \\ \text { New Project }\end{array} & \begin{array}{l}\text { Ongoing Project } \\ \text { Life (in years) }\end{array} \\ \text { Recycle/Reuse HAZ } & \text { Routine } & \text { Ongoing } & 1\end{array}$

Waste Reduction

\begin{tabular}{|l|l|l|l||l|}
\hline Quarter 1 & Quarter 2 & Quarter 3 & Quarter 4 & YTD Reduction \\
\hline $0.07 \mathrm{mt}$ & $0.07 \mathrm{mt}$ & $0.00 \mathrm{mt}$ & $0.00 \mathrm{mt}$ & $0.14 \mathrm{mt}$ \\
\hline
\end{tabular}

\section{Savings}

\begin{tabular}{|l|l|l|l|l|}
\hline Quarter 1 & Quarter 2 & Quarter 3 & Quarter 4 & YTD Savings \\
\hline $4,900.00$ & $4,760.00$ & 0.00 & 0.00 & $9,660.00$ \\
\hline
\end{tabular}

Accomplishment: Aerosol cans are redeployed onsite.

\section{Hanford Site}

$\begin{array}{lllll}\text { P2 Activity } & \text { Waste Type } & \begin{array}{l}\text { Routine or } \\ \text { Cleanup/Stabilization }\end{array} & \begin{array}{l}\text { Ongoing Project or } \\ \text { New Project }\end{array} & \begin{array}{l}\text { Ongoing Project } \\ \text { Life (in years) }\end{array} \\ \text { Recycle/Reuse HAZ } & \text { Cleanup/Stabilization } & \text { Ongoing } & 1\end{array}$

This accomplishment counts toward the DOE-wide reduction goal (i.e. 10\%) for waste resulting from cleanup, stabilization, and decommissioning activities.

Waste Reduction

\begin{tabular}{|c|c|c|c|c|}
\hline Quarter 1 & Quarter 2 & Quarter 3 & Quarter 4 & YTD Reduction \\
\hline $0.10 \mathrm{mt}$ & $0.00 \mathrm{mt}$ & $0.00 \mathrm{mt}$ & $0.00 \mathrm{mt}$ & $0.10 \mathrm{mt}$ \\
\hline \multicolumn{5}{|c|}{ Savings } \\
\hline Quarter 1 & Quarter 2 & Quarter 3 & Quarter 4 & YTD Savings \\
\hline 0.00 & 0.00 & 0.00 & 0.00 & 0.00 \\
\hline
\end{tabular}

Accomplishment: Chemicals were redeployed to the Port of Ephrata and to St Joseph School in Kennewick 


\section{Hanford Site}

$\begin{array}{lllll}\text { P2 Activity Waste Type } & \begin{array}{l}\text { Routine or } \\ \text { Cleanup/Stabilization }\end{array} & \begin{array}{l}\text { Ongoing Project or } \\ \text { New Project }\end{array} & \begin{array}{l}\text { Ongoing Project } \\ \text { Life (in years) }\end{array} \\ \text { Recycle/Reuse HAZ } & \text { Routine } & \text { Ongoing } & 1\end{array}$

Waste Reduction

\begin{tabular}{|c|c|c|c|c|}
\hline Quarter 1 & Quarter 2 & Quarter 3 & Quarter 4 & YTD Reduction \\
\hline $7.80 \mathrm{mt}$ & $0.00 \mathrm{mt}$ & $6.72 \mathrm{mt}$ & $15.38 \mathrm{mt}$ & $29.90 \mathrm{mt}$ \\
\hline \multicolumn{5}{|c|}{ Savings } \\
\hline Quarter 1 & Quarter 2 & Quarter 3 & Quarter 4 & YTD Savings \\
\hline $12,324.00$ & 0.00 & $10,614.00$ & $12,796.00$ & $35,734.00$ \\
\hline
\end{tabular}

Accomplishment: Excess bulk lead (bricks, sheets, etc.) is collected and sent to an off-site recycler.

\section{Hanford Site}

$\begin{array}{lllll}\text { P2 Activity } & \text { Waste Type } & \begin{array}{l}\text { Routine or } \\ \text { Cleanup/Stabilization }\end{array} & \begin{array}{l}\text { Ongoing Project or } \\ \text { New Project }\end{array} & \begin{array}{l}\text { Ongoing Project } \\ \text { Life (in years) }\end{array} \\ \text { Recycle/Reuse HAZ } & - & \text { Cleanup/Stabilization } & \text { Ongoing } & 1\end{array}$

This accomplishment counts toward the DOE-wide reduction goal (i.e. 10\%) for waste resulting from cleanup, stabilization, and decommissioning activities.

Waste Reduction

\begin{tabular}{|l|l|l|l|l|}
\hline Quarter 1 & Quarter 2 & Quarter 3 & Quarter 4 & YTD Reduction \\
\hline \hline $0.00 \mathrm{mt}$ & $1.00 \mathrm{mt}$ & $0.00 \mathrm{mt}$ & $0.00 \mathrm{mt}$ & $1.00 \mathrm{mt}$ \\
\hline \multicolumn{3}{|c|}{ Savings } \\
\hline Quarter 1 & Quarter 2 & Quarter 3 & Quarter 4 & YTD Savings \\
\hline \hline 0.00 & $11,700.00$ & 0.00 & 0.00 & $11,700.00$ \\
\hline
\end{tabular}

Accomplishment: Four drums of $93 \%$ sulfuric acid that was identified as unneeded was used as a $\mathrm{pH}$ stabilizer at HR-3 and KR-4 Operable Unit pump and treat units.

\section{Hanford Site}

$\begin{array}{lllll}\text { P2 Activity Waste Type } & \begin{array}{l}\text { Routine or } \\ \text { Cleanup/Stabilization }\end{array} & \begin{array}{l}\text { Ongoing Project or } \\ \text { New Project }\end{array} & \begin{array}{l}\text { Ongoing Project } \\ \text { Life (in years) }\end{array} \\ \text { Recycle/Reuse HAZ } & \text { Cleanup/Stabilization } & \text { Ongoing } & 1\end{array}$


This accomplishment counts toward the DOE-wide reduction goal (i.e. 10\%) for waste resulting from cleanup, stabilization, and decommissioning activities.

\begin{tabular}{|c|c|c|c|c|}
\hline \multicolumn{5}{|c|}{ Waste Reduction } \\
\hline Quarter 1 & Quarter 2 & Quarter 3 & Quarter 4 & YTD Reduction \\
\hline $8.20 \mathrm{mt}$ & $0.00 \mathrm{mt}$ & $0.00 \mathrm{mt}$ & $0.00 \mathrm{mt}$ & $8.20 \mathrm{mt}$ \\
\hline \multicolumn{5}{|c|}{ Savings } \\
\hline Quarter 1 & Quarter 2 & Quarter 3 & Quarter 4 & YTD Savings \\
\hline $93,440.00$ & 0.00 & 0.00 & 0.00 & $93,440.00$ \\
\hline
\end{tabular}

Accomplishment: Granulated activated carbon was sent off site and regenerated.

\section{Hanford Site}

\begin{tabular}{|c|c|c|c|c|}
\hline P2 Activity & Waste Type & $\begin{array}{l}\text { Routine or } \\
\text { Cleanup/Stabilization }\end{array}$ & $\begin{array}{l}\text { Ongoing Project or } \\
\text { New Project }\end{array}$ & $\begin{array}{l}\text { Ongoing Project } \\
\text { Life (in years) }\end{array}$ \\
\hline Recycle/Reus & $\mathrm{HAZ}$ & Routine & Ongoing & 1 \\
\hline
\end{tabular}

Waste Reduction

\begin{tabular}{|l|l|l|l|l|}
\hline Quarter 1 & Quarter 2 & Quarter 3 & Quarter 4 & YTD Reduction \\
\hline \hline $0.88 \mathrm{mt}$ & $0.00 \mathrm{mt}$ & $0.03 \mathrm{mt}$ & $0.48 \mathrm{mt}$ & $1.39 \mathrm{mt}$ \\
\hline & \multicolumn{2}{|c|}{ Savings } \\
\hline \multicolumn{1}{|l|}{ Quarter 1 } & Quarter 2 & Quarter 3 & Quarter 4 & YTD Savings \\
\hline $2,443.00$ & 0.00 & 61.00 & $10,442.00$ & $12,946.00$ \\
\hline
\end{tabular}

Accomplishment: Incandescent bulbs (First Quarter: 0.08 metric tons; Third Quarter: 0.01 metric tons), sodium vapor bulbs (First Quarter: 0.72 metric tons; Fourth Quarter: 0.32 metric tons), and mercury vapor bulbs (First Quarter: 0.08 metric tons; Third Quarter: 0.014 metric tons; Fourth Quarter $0.16 \mathrm{mt}$ ) are shipped to an off-site recycler.

\section{Hanford Site}

\begin{tabular}{|c|c|c|c|c|}
\hline P2 Activity & Waste Type & $\begin{array}{l}\text { Routine or } \\
\text { Cleanup/Stabilization }\end{array}$ & $\begin{array}{l}\text { Ongoing Project or } \\
\text { New Project }\end{array}$ & $\begin{array}{l}\text { Ongoing Project } \\
\text { Life (in years) }\end{array}$ \\
\hline Recycle/Reus & HAZ & Routine & Ongoing & 1 \\
\hline
\end{tabular}

Waste Reduction

\begin{tabular}{|l|l|l|l|l|}
\hline Quarter 1 & Quarter 2 & Quarter 3 & Quarter 4 & YTD Reduction \\
\hline $2.34 \mathrm{mt}$ & $1.82 \mathrm{mt}$ & $34.76 \mathrm{mt}$ & $0.77 \mathrm{mt}$ & $39.69 \mathrm{mt}$ \\
\hline
\end{tabular}


Savings

\begin{tabular}{|l|l|l|l|l|}
\hline Quarter 1 & Quarter 2 & Quarter 3 & Quarter 4 & YTD Savings \\
\hline \hline 777.00 & 602.00 & $53,938.00$ & $16,677.00$ & $71,994.00$ \\
\hline
\end{tabular}

Accomplishment: Lead-acid batteries are recycled through a closed-loop contract or shipped offsite for recycling, and miscellaneous small batteries are shipped offsite for recycling.

\section{Hanford Site}

$\begin{array}{llll}\text { P2 Activity Waste Type } & \begin{array}{l}\text { Routine or } \\ \text { Cleanup/Stabilization }\end{array} & \begin{array}{l}\text { Ongoing Project or } \\ \text { New Project }\end{array} & \begin{array}{l}\text { Ongoing Project } \\ \text { Life (in years) }\end{array} \\ \text { Recycle/Reuse HAZ } & \text { Routine } & \text { Ongoing } & 1\end{array}$

Waste Reduction

\begin{tabular}{|l|l|l|l|l|}
\hline Quarter 1 & Quarter 2 & Quarter 3 & Quarter 4 & YTD Reduction \\
\hline \hline $9.59 \mathrm{mt}$ & $1.70 \mathrm{mt}$ & $2.84 \mathrm{mt}$ & $2.00 \mathrm{mt}$ & $16.13 \mathrm{mt}$ \\
\hline
\end{tabular}

Savings

\begin{tabular}{|l|l|l|l|l|}
\hline \hline Quarter 1 & Quarter 2 & Quarter 3 & Quarter 4 & YTD Savings \\
\hline $1,198.00$ & $8,490.00$ & $14,180.00$ & $43,600.00$ & $67,468.00$ \\
\hline
\end{tabular}

Accomplishment: Machine coolants are recycled within the Fabrication Services organization.

\section{Hanford Site}

$\begin{array}{llll}\text { P2 Activity Waste Type } & \begin{array}{l}\text { Routine or } \\ \text { Cleanup/Stabilization }\end{array} & \begin{array}{l}\text { Ongoing Project or } \\ \text { New Project }\end{array} & \begin{array}{l}\text { Ongoing Project } \\ \text { Life (in years) }\end{array} \\ \text { Recycle/Reuse HAZ } & \text { Routine } & \text { Ongoing } & 1\end{array}$

Waste Reduction

\begin{tabular}{|c|c|c|c|c|}
\hline Quarter 1 & Quarter 2 & Quarter 3 & Quarter 4 & YTD Reduction \\
\hline $0.25 \mathrm{mt}$ & $0.23 \mathrm{mt}$ & $0.14 \mathrm{mt}$ & $0.32 \mathrm{mt}$ & $0.94 \mathrm{mt}$ \\
\hline \multicolumn{5}{|c|}{ Savings } \\
\hline Quarter 1 & Quarter 2 & Quarter 3 & Quarter 4 & YTD Savings \\
\hline $3,206.00$ & $2,913.00$ & $1,112.00$ & $6,911.00$ & $14,142.00$ \\
\hline
\end{tabular}

Accomplishment: Shop towels are provided through a closed-loop contract that returns the towels to the vendor for laundering. 


\section{Hanford Site}

$\begin{array}{lllll}\text { P2 Activity } & \text { Waste Type } & \begin{array}{l}\text { Routine or } \\ \text { Cleanup/Stabilization }\end{array} & \begin{array}{l}\text { Ongoing Project or } \\ \text { New Project }\end{array} & \begin{array}{l}\text { Ongoing Project } \\ \text { Life (in years) }\end{array} \\ \text { Recycle/Reuse SAN } & \text { Cleanup/Stabilization } & \text { Ongoing } & 1\end{array}$

This accomplishment counts toward the DOE-wide reduction goal (i.e. 10\%) for waste resulting from cleanup, stabilization, and decommissioning activities.

Waste Reduction

\begin{tabular}{|l|l|l|l|l|}
\hline Quarter 1 & Quarter 2 & Quarter 3 & Quarter 4 & YTD Reduction \\
\hline $0.53 \mathrm{mt}$ & $0.00 \mathrm{mt}$ & $0.00 \mathrm{mt}$ & $0.00 \mathrm{mt}$ & $0.53 \mathrm{mt}$ \\
\hline
\end{tabular}

Accomplishment: 57 unneeded 90 mil liners stored at the $1330-\mathrm{N}$ waste pad were given to Waste Management Federal Services of Hanford, Inc. for use.

\section{Hanford Site}

$\begin{array}{lllll}\text { P2 Activity } & \text { Waste Type } & \begin{array}{l}\text { Routine or } \\ \text { Cleanup/Stabilization }\end{array} & \begin{array}{l}\text { Ongoing Project or } \\ \text { New Project }\end{array} & \begin{array}{l}\text { Ongoing Project } \\ \text { Life (in years) }\end{array} \\ \text { Recycle/Reuse SAN } & \text { Routine } & \text { Ongoing } & 1\end{array}$

Waste Reduction

\begin{tabular}{|l|l|l|l|l|}
\hline Quarter 1 & Quarter 2 & Quarter 3 & Quarter 4 & YTD Reduction \\
\hline $11.04 \mathrm{mt}$ & $13.71 \mathrm{mt}$ & $10.93 \mathrm{mt}$ & $15.59 \mathrm{mt}$ & $51.27 \mathrm{mt}$ \\
\hline \multicolumn{3}{|c|}{ Savings } \\
\hline \multicolumn{1}{|c|}{ Quarter 1 } & Quarter 2 & Quarter 3 & Quarter 4 & YTD Savings \\
\hline $1,435.00$ & $1,782.00$ & $1,421.00$ & $12,970.00$ & $17,608.00$ \\
\hline
\end{tabular}

Accomplishment: Cardboard collected from Project Hanford Management Contractors is recycled by an offsite vendor. 


\section{Hanford Site}

\begin{tabular}{|c|c|c|c|c|}
\hline P2 Activity & Waste Type & $\begin{array}{l}\text { Routine or } \\
\text { Cleanup/Stabilization }\end{array}$ & $\begin{array}{l}\text { Ongoing Project or } \\
\text { New Project }\end{array}$ & $\begin{array}{l}\text { Ongoing Project } \\
\text { Life (in years) }\end{array}$ \\
\hline Recycle/Reu & SAN & Cleanup/Stabilization & Ongoing & 1 \\
\hline
\end{tabular}

This accomplishment counts toward the DOE-wide reduction goal (i.e. 10\%) for waste resulting from cleanup, stabilization, and decommissioning activities.

Waste Reduction

\begin{tabular}{|l|l|l||l|l|}
\hline Quarter 1 & Quarter 2 & Quarter 3 & Quarter 4 & YTD Reduction \\
\hline \hline $5.00 \mathrm{mt}$ & $0.00 \mathrm{mt}$ & $0.00 \mathrm{mt}$ & $0.00 \mathrm{mt}$ & $5.00 \mathrm{mt}$ \\
\hline
\end{tabular}

Savings

\begin{tabular}{|l|l|l|l|l|}
\hline Quarter 1 & Quarter 2 & Quarter 3 & Quarter 4 & YTD Savings \\
\hline \hline $31,080.00$ & 0.00 & 0.00 & 0.00 & $31,080.00$ \\
\hline
\end{tabular}

Accomplishment: Dow X21 resin was sent off site and regenerated.

\section{Hanford Site}

$\begin{array}{llll}\text { P2 Activity Waste Type } & \begin{array}{l}\text { Routine or } \\ \text { Cleanup/Stabilization }\end{array} & \begin{array}{l}\text { Ongoing Project or } \\ \text { New Project }\end{array} & \begin{array}{l}\text { Ongoing Project } \\ \text { Life (in years) }\end{array} \\ \text { Recycle/Reuse SAN } & \text { Routine } & \text { Ongoing } & 1\end{array}$

Waste Reduction

\begin{tabular}{|l|l|l|l|l|}
\hline Quarter 1 & Quarter 2 & Quarter 3 & Quarter 4 & YTD Reduction \\
\hline \hline $179.73 \mathrm{mt}$ & $140.09 \mathrm{mt}$ & $96.56 \mathrm{mt}$ & $131.69 \mathrm{mt}$ & $548.07 \mathrm{mt}$ \\
\hline
\end{tabular}

\section{Savings}

\begin{tabular}{|l|l|l|l|l|}
\hline Quarter 1 & Quarter 2 & Quarter 3 & Quarter 4 & YTD Savings \\
\hline $52,161.00$ & $44,761.00$ & $27,956.00$ & $109,567.00$ & $234,445.00$ \\
\hline
\end{tabular}

Accomplishment: Ferrous metals(First Quarter: 160.95 metric tons; Second Quarter: 115.00 metric tons; Third Quarter: 86.69 metric tons; Fourth Quarter: 115.10 metric tons), or non-ferrous metals (First Quarter: 18.59 metric tons; Second Quarter: 25.00 metric tons; Third Quarter: 9.80 metric tons; Fourth Quarter: 16.53 metric tons) from Bechtel Hanford Incorporated, Project Hanford Management Contract and Pacific Northwest National Laboratory are sold to a local recycler. Tin (First Quarter: 0.19 metric ton; Second Quarter: 0.088 metric tons; Third Quarter: 0.061 metric tons; Fourth Quarter: 0.061 metric tons) from Bechtel Hanford, Inc. and Project Hanford Management Contract is recycled through an offsite vendor. 


\section{Hanford Site}

\begin{tabular}{|c|c|c|c|c|}
\hline P2 Activity & Waste Type & $\begin{array}{l}\text { Routine or } \\
\text { Cleanup/Stabilization }\end{array}$ & $\begin{array}{l}\text { Ongoing Project or } \\
\text { New Project }\end{array}$ & $\begin{array}{l}\text { Ongoing Project } \\
\text { Life (in years) }\end{array}$ \\
\hline Recycle/Reu: & $\mathrm{SAN}$ & Routine & Ongoing & 1 \\
\hline
\end{tabular}

Waste Reduction

\begin{tabular}{|l|l|l|l|l|}
\hline Quarter 1 & Quarter 2 & Quarter 3 & Quarter 4 & YTD Reduction \\
\hline $0.41 \mathrm{mt}$ & $0.28 \mathrm{mt}$ & $0.22 \mathrm{mt}$ & $0.19 \mathrm{mt}$ & $1.10 \mathrm{mt}$ \\
\hline
\end{tabular}

\section{Savings}

\begin{tabular}{|l|l|l|l|l|}
\hline Quarter 1 & Quarter 2 & Quarter 3 & Quarter 4 & YTD Savings \\
\hline 53.00 & 36.00 & 28.00 & 160.00 & 277.00 \\
\hline
\end{tabular}

Accomplishment: Glass from Bechtel Hanford, Inc. and Project Hanford Management Contractors is recycled through an offsite vendor.

\section{Hanford Site}

$\begin{array}{lllll}\text { P2 Activity } & \text { Waste Type } & \text { Routine or } & \text { Ongoing Project or } & \text { Ongoing Project } \\ & \cdot & \text { Cleanup/Stabilization } & \text { New Project } & \text { Life (in years) } \\ \text { Recycle/Reuse SAN } & \text { Routine } & \text { Ongoing } & 1\end{array}$

Waste Reduction

\begin{tabular}{|l|l|l|l|l|}
\hline Quarter 1 & Quarter 2 & Quarter 3 & Quarter 4 & YTD Reduction \\
\hline $0.22 \mathrm{mt}$ & $0.00 \mathrm{mt}$ & $0.00 \mathrm{mt}$ & $0.45 \mathrm{mt}$ & $0.67 \mathrm{mt}$ \\
\hline
\end{tabular}

\section{Savings}

\begin{tabular}{|l|l|l|l|l|}
\hline Quarter 1 & Quarter 2 & Quarter 3 & Quarter 4 & YTD Savings \\
\hline 28.00 & 0.00 & 0.00 & $9,435.60$ & $9,463.60$ \\
\hline
\end{tabular}

Accomplishment: Halon and General A, B, C fire extinguishers are shipped to the 1162 Building to be sent offsite for refurbishining (Maintenance and Testing) and returned to the site for reuse. During the Fourth Quarter, excessed 56 additional fire extinguishers.

\section{Hanford Site}

\begin{tabular}{|c|c|c|c|c|}
\hline P2 Activity & Waste Type & $\begin{array}{l}\text { Routine or } \\
\text { Cleanup/Stabilization }\end{array}$ & $\begin{array}{l}\text { Ongoing Project or } \\
\text { New Project }\end{array}$ & $\begin{array}{l}\text { Ongoing Project } \\
\text { Life (in years) }\end{array}$ \\
\hline Recycle/Reus & SAN & Routine & Ongoing & 1 \\
\hline
\end{tabular}


Waste Reduction

\begin{tabular}{|c|c|c|c|c|}
\hline Quarter 1 & Quarter 2 & Quarter 3 & Quarter 4 & YTD Reduction \\
\hline $3.49 \mathrm{mt}$ & $0.00 \mathrm{mt}$ & $2.23 \mathrm{mt}$ & $4.48 \mathrm{mt}$ & $10.20 \mathrm{mt}$ \\
\hline \multicolumn{5}{|c|}{ Savings } \\
\hline Quarter 1 & Quarter 2 & Quarter 3 & Quarter 4 & YTD Savings \\
\hline 419.00 & 0.00 & 267.00 & $3,720.00$ & $4,406.00$ \\
\hline
\end{tabular}

Accomplishment: Intact and crushed fluorescent tubes are shipped to an off-site recycler. Reported values include recycling efforts by Bechtel Hanford Incorporated, Project Hanford Management Contract, and Pacific Northwest National Laboratory.

\section{Hanford Site}

$\begin{array}{lllll}\text { P2 Activity } & \text { Waste Type } & \begin{array}{l}\text { Routine or } \\ \text { Cleanup/Stabilization }\end{array} & \begin{array}{l}\text { Ongoing Project or } \\ \text { New Project } \\ \text { Ongoing }\end{array} & \begin{array}{l}\text { Ongoing Project } \\ \text { Life (in years) }\end{array} \\ \text { Recycle/Reuse SAN } & \text { Routine } & \text { Ongoing } & 1\end{array}$

Waste Reduction

\begin{tabular}{|c|c|c|c|c|}
\hline Quarter 1 & Quarter 2 & Quarter 3 & Quarter 4 & YTD Reduction \\
\hline $3.57 \mathrm{mt}$ & $4.53 \mathrm{mt}$ & $5.07 \mathrm{mt}$ & $5.25 \mathrm{mt}$ & $18.42 \mathrm{mt}$ \\
\hline \multicolumn{5}{|c|}{ Savings } \\
\hline Quarter 1 & Quarter 2 & Quarter 3 & Quarter 4 & YTD Savings \\
\hline 464.00 & 589.00 & 659.00 & $4,368.00$ & $6,080.00$ \\
\hline
\end{tabular}

Accomplishment: Newsprint and Magazines are recycled through an offsite vendor.

\section{Hanford Site}

$\begin{array}{lllll}\text { P2 Activity } & \text { Waste Type } & \begin{array}{l}\text { Routine or } \\ \text { Cleanup/Stabilization }\end{array} & \begin{array}{l}\text { Ongoing Project or } \\ \text { New Project }\end{array} & \begin{array}{l}\text { Ongoing Project } \\ \text { Life (in years) }\end{array} \\ \text { Recycle/Reuse SAN } & \text { Routine } & \text { Ongoing } & 1\end{array}$

Waste Reduction

\begin{tabular}{|c|c|c|c|c|}
\hline Quarter 1 & Quarter 2 & Quarter 3 & Quarter 4 & YTD Reduction \\
\hline $0.12 \mathrm{mt}$ & $0.00 \mathrm{mt}$ & $1.42 \mathrm{mt}$ & $2.82 \mathrm{mt}$ & $4.36 \mathrm{mt}$ \\
\hline \multicolumn{5}{|c|}{ Savings } \\
\hline Quarter 1 & Quarter 2 & Quarter 3 & Quarter 4 & YTD Savings \\
\hline 16.00 & 0.00 & 85.00 & $\mid 2,340.00$ & $2,441.00$ \\
\hline
\end{tabular}


Accomplishment: Non-polychlorinated biphenyl light ballasts are sent to a recycler for recovery of metals.

\section{Hanford Site}

$\begin{array}{lllll}\text { P2 Activity } & \text { Waste Type } & \begin{array}{l}\text { Routine or } \\ \text { Cleanup/Stabilization }\end{array} & \begin{array}{l}\text { Ongoing Project or } \\ \text { New Project }\end{array} & \begin{array}{l}\text { Ongoing Project } \\ \text { Life (in years) }\end{array} \\ \text { Recycle/Reuse SAN } & \text { Routine } & \text { Ongoing } & 1\end{array}$

Waste Reduction

\begin{tabular}{|l|l|l|l|l|}
\hline Quarter 1 & Quarter 2 & Quarter 3 & Quarter 4 & YTD Reduction \\
\hline $100.80 \mathrm{mt}$ & $90.32 \mathrm{mt}$ & $97.69 \mathrm{mt}$ & $105.54 \mathrm{mt}$ & $394.35 \mathrm{mt}$ \\
\hline
\end{tabular}

Savings

\begin{tabular}{|l|l|l|l|l|}
\hline Quarter 1 & Quarter 2 & Quarter 3 & Quarter 4 & YTD Savings \\
\hline $13,104.00$ & $11,742.00$ & $12,670.00$ & $87,809.00$ & $125,325.00$ \\
\hline
\end{tabular}

Accomplishment: Paper from Bechtel Hanford Incorporated and Project Hanford Management Contract is sold to a local recycler.

\section{Hanford Site}

\begin{tabular}{|c|c|c|c|c|}
\hline P2 Activity & Waste Type & $\begin{array}{l}\text { Routine or } \\
\text { Cleanup/Stabilization }\end{array}$ & $\begin{array}{l}\text { Ongoing Project or } \\
\text { New Project }\end{array}$ & $\begin{array}{l}\text { Ongoing Project } \\
\text { Life (in years) }\end{array}$ \\
\hline Recycle/Reus & SAN & Routine & Ongoing & 1 \\
\hline
\end{tabular}

Waste Reduction

\begin{tabular}{|l|l|l|l|l|}
\hline Quarter 1 & Quarter 2 & Quarter 3 & Quarter 4 & YTD Reduction \\
\hline $0.10 \mathrm{mt}$ & $0.04 \mathrm{mt}$ & $0.24 \mathrm{mt}$ & $0.21 \mathrm{mt}$ & $0.59 \mathrm{mt}$ \\
\hline & \multicolumn{2}{|c|}{ Savings } \\
\hline
\end{tabular}

Accomplishment: Plastics are recycled through an offsite vendor. 


\section{Hanford Site}

\begin{tabular}{|c|c|c|c|c|}
\hline P2 Activity & Waste Type & $\begin{array}{l}\text { Routine or } \\
\text { Cleanup/Stabilization }\end{array}$ & $\begin{array}{l}\text { Ongoing Project or } \\
\text { New Project }\end{array}$ & $\begin{array}{l}\text { Ongoing Project } \\
\text { Life (in years) }\end{array}$ \\
\hline Recycle/Reu & SAN & Routine & Ongoing & 1 \\
\hline
\end{tabular}

Waste Reduction

\begin{tabular}{|c|c|c|c|c|}
\hline Quarter 1 & Quarter 2 & Quarter 3 & Quarter 4 & YTD Reduction \\
\hline $0.00 \mathrm{mt}$ & $1.46 \mathrm{mt}$ & $0.21 \mathrm{mt}$ & $2.25 \mathrm{mt}$ & $3.92 \mathrm{mt}$ \\
\hline \multicolumn{5}{|c|}{ Savings ... } \\
\hline Quarter 1 & Quarter 2 & Quarter 3 & Quarter 4 & YTD Savings \\
\hline 0.00 & 189.00 & 26.00 & $1,868.00$ & $2,083.00$ \\
\hline
\end{tabular}

Accomplishment: Propylene Glycol is recycled and reused on the site. During the Fourth Quarter the Propylene Glycol was sold at Public Auction.

\section{Hanford Site}

$\begin{array}{llll}\text { P2 Activity Waste Type } & \begin{array}{l}\text { Routine or } \\ \text { Cleanup/Stabilization }\end{array} & \begin{array}{l}\text { Ongoing Project or } \\ \text { New Project }\end{array} & \begin{array}{l}\text { Ongoing Project } \\ \text { Life (in years) }\end{array} \\ \text { Recycle/Reuse SAN } & \text { Routine } & \text { Ongoing } & 1\end{array}$

Waste Reduction

\begin{tabular}{|l|l|l|l|l|}
\hline Quarter 1 & Quarter 2 & Quarter 3 & Quarter 4 & YTD Reduction \\
\hline \hline $4.87 \mathrm{mt}$ & $2.12 \mathrm{mt}$ & \multicolumn{1}{|c|}{ Savings } & $11.70 \mathrm{mt}$ \\
\hline \multicolumn{3}{|c|}{} & \multicolumn{1}{|c|}{ S.12 mt } \\
\hline Quarter 1 & Quarter 2 & Quarter 3 & Quarter 4 & YTD Savings \\
\hline $4,869.00$ & $1,912.00$ & $2,590.00$ & $1,755.00$ & $11,126.00$ \\
\hline
\end{tabular}

Accomplishment: Tires are sent offsite for retreading through a closed loop contract.

\section{Hanford Site}

$\begin{array}{llll}\text { P2 Activity Waste Type } & \begin{array}{l}\text { Routine or } \\ \text { Cleanup/Stabilization }\end{array} & \begin{array}{l}\text { Ongoing Project or } \\ \text { New Project }\end{array} & \begin{array}{l}\text { Ongoing Project } \\ \text { Life (in years) }\end{array} \\ \text { Recycle/Reuse SAN } & \text { Routine } & \text { Ongoing } & 1\end{array}$




\begin{tabular}{|l|l|l|l|l|}
\hline Quarter 1 & Quarter 2 & Quarter 3 & Quarter 4 & YTD Reduction \\
\hline $3.39 \mathrm{mt}$ & $2.72 \mathrm{mt}$ & $3.05 \mathrm{mt}$ & $2.97 \mathrm{mt}$ & $12.13 \mathrm{mt}$ \\
\hline
\end{tabular}

\section{Savings}

\begin{tabular}{|l|l|l|l|l|}
\hline Quarter 1 & Quarter 2 & Quarter 3 & Quarter 4 & YTD Savings \\
\hline \hline 678.00 & 544.00 & 610.00 & $2,471.00$ & $4,303.00$ \\
\hline
\end{tabular}

Accomplishment: Toner cartridges are sent to a local contractor for recycling.

\section{Hanford Site}

\begin{tabular}{|c|c|c|c|c|}
\hline P2 Activity & Waste Type & $\begin{array}{l}\text { Routine or } \\
\text { Cleanup/Stabilization }\end{array}$ & $\begin{array}{l}\text { Ongoing Project or } \\
\text { New Project }\end{array}$ & $\begin{array}{l}\text { Ongoing Project } \\
\text { Life (in years) }\end{array}$ \\
\hline Recycle/Ret & & Cleanup/Stabilization & Ongoing & 1 \\
\hline
\end{tabular}

This accomplishment counts toward the DOE-wide reduction goal (i.e. 10\%) for waste resulting from cleanup, stabilization, and decommissioning activities.

Waste Reduction

\begin{tabular}{|c|c|c|c|c|}
\hline Quarter 1 & Quarter 2 & Quarter 3 & Quarter 4 & YTD Reduction \\
\hline $0.20 \mathrm{mt}$ & $0.20 \mathrm{mt}$ & $0.20 \mathrm{mt}$ & $0.45 \mathrm{mt}$ & $1.05 \mathrm{mt}$ \\
\hline \multicolumn{5}{|c|}{ Savings } \\
\hline Quarter 1 & Quarter 2 & Quarter 3 & Quarter 4 & YTD Savings \\
\hline $9,779.00$ & $10,160.00$ & $7,470.00$ & $12,259.00$ & $39,668.00$ \\
\hline
\end{tabular}

Accomplishment: Toner cartridges returned to vendor for recycle.

\section{Hanford Site}

$\begin{array}{lllll}\text { P2 Activity } & \text { Waste Type } & \begin{array}{l}\text { Routine or } \\ \text { Cleanup/Stabilization }\end{array} & \begin{array}{l}\text { Ongoing Project or } \\ \text { New Project }\end{array} & \begin{array}{l}\text { Ongoing Project } \\ \text { Life (in years) }\end{array} \\ \text { Recycle/Reuse SAN } & \text { Routine } & \text { Ongoing } & 1\end{array}$

Waste Reduction

\begin{tabular}{|c|c|c|c|c|}
\hline Quarter 1 & Quarter 2 & Quarter 3 & Quarter 4 & YTD Reduction \\
\hline $3.11 \mathrm{mt}$ & $6.30 \mathrm{mt}$ & $2.99 \mathrm{mt}$ & $2.34 \mathrm{mt}$ & $14.74 \mathrm{mt}$ \\
\hline \multicolumn{5}{|c|}{ Savings } \\
\hline Quarter 1 & Quarter 2 & Quarter 3 & Quarter 4 & YTD Savings \\
\hline 404.00 & 819.00 & 389.00 & $1,946.00$ & $3,558.00$ \\
\hline
\end{tabular}

Accomplishment: Unusable software is sent to a plastics recycler. 


\section{Hanford Site}

$\begin{array}{llll}\text { P2 Activity Waste Type } & \begin{array}{l}\text { Routine or } \\ \text { Cleanup/Stabilization }\end{array} & \begin{array}{l}\text { Ongoing Project or } \\ \text { New Project }\end{array} & \begin{array}{l}\text { Ongoing Project } \\ \text { Life (in years) }\end{array} \\ \text { Recycle/Reuse SAN } & \text { Routine } & \text { Ongoing } & 1\end{array}$

Waste Reduction .

\begin{tabular}{|l|l|l|l|l||}
\hline Quarter 1 & Quarter 2 & Quarter 3 & Quarter 4 & YTD Reduction \\
\hline \hline $25.80 \mathrm{mt}$ & $0.00 \mathrm{mt}$ & $0.00 \mathrm{mt}$ & $7.28 \mathrm{mt}$ & $33.08 \mathrm{mt}$ \\
\hline \multicolumn{2}{|c|}{ Savings } \\
\hline \multicolumn{1}{|c|}{ Quarter 1 } & Quarter 2 & Quarter 3 & Quarter 4 & YTD Savings \\
\hline \hline $6,450.00$ & 0.00 & 0.00 & $6,057.00$ & $12,507.00$ \\
\hline
\end{tabular}

Accomplishment: Used furniture and appliances are sent to a recycler.

\section{Hanford Site}

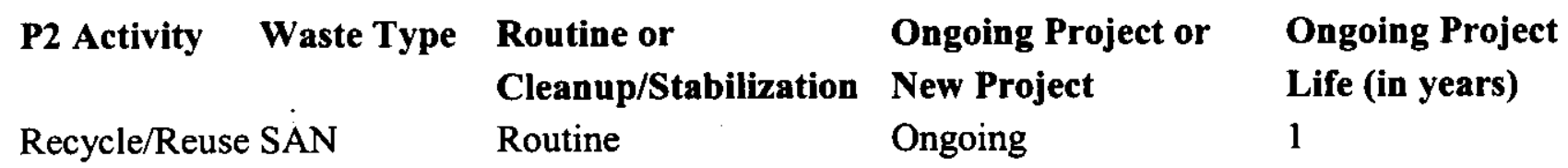

Waste Reduction

\begin{tabular}{||l|l|l|l|l||}
\hline Quarter 1 & Quarter 2 & Quarter 3 & Quarter 4 & YTD Reduction \\
\hline \hline $7.82 \mathrm{mt}$ & $5.77 \mathrm{mt}$ & $8.30 \mathrm{mt}$ & $12.15 \mathrm{mt}$ & $34.04 \mathrm{mt}$ \\
\hline
\end{tabular}

Savings

\begin{tabular}{|l|l|l|l|l||}
\hline Quarter 1 & Quarter 2 & Quarter 3 & Quarter 4 & YTD Savings \\
\hline \hline $1,564.00$ & $1,154.00$ & $1,660.00$ & $10,109.00$ & $14,487.00$ \\
\hline
\end{tabular}

Accomplishment: Used oil is returned to a vendor for re-refining through a closed loop contract.

\section{Hanford Site}

\begin{tabular}{|c|c|c|c|c|}
\hline P2 Activity & Waste Type & $\begin{array}{l}\text { Routine or } \\
\text { Cleanup/Stabilization }\end{array}$ & $\begin{array}{l}\text { Ongoing Project or } \\
\text { New Project }\end{array}$ & $\begin{array}{l}\text { Ongoing Project } \\
\text { Life (in years) }\end{array}$ \\
\hline Recycle/Reus & SAN & Routine & Ongoing & 1 \\
\hline
\end{tabular}

Waste Reduction 


\begin{tabular}{|l|l|l|l|l|}
\hline Quarter 1 & Quarter 2 & Quarter 3 & Quarter 4 & YTD Reduction \\
\hline \hline $0.00 \mathrm{mt}$ & $1.08 \mathrm{mt}$ & $64.12 \mathrm{mt}$ & $54.96 \mathrm{mt}$ & $120.16 \mathrm{mt}$ \\
\hline
\end{tabular}

\section{Savings}

\begin{tabular}{|l|l|l|l|l|}
\hline Quarter 1 & Quarter 2 & Quarter 3 & Quarter 4 & YTD Savings \\
\hline \hline 0.00 & 140.00 & $8,726.00$ & $46,559.00$ & $55,425.00$ \\
\hline
\end{tabular}

Accomplishment: Wood is sent to a local landfill or to cascade Pallet Inc. where it is chipped and used for landscaping and other purposes.

\section{Hanford Site}

$\begin{array}{lllll}\text { P2 Activity } & \text { Waste Type } & \begin{array}{l}\text { Routine or } \\ \text { Cleanup/Stabilization }\end{array} & \begin{array}{l}\text { Ongoing Project or } \\ \text { New Project }\end{array} & \begin{array}{l}\text { Ongoing Project } \\ \text { Life (in years) }\end{array} \\ \begin{array}{l}\text { Source } \\ \text { Reduction }\end{array} & \text { LLW } & \text { Cleanup/Stabilization } & \text { Ongoing } & 1\end{array}$

This accomplishment counts toward the DOE-wide reduction goal (i.e. 10\%) for waste resulting from cleanup, stabilization, and decommissioning activities.

Waste Reduction

\begin{tabular}{|c|c|c|c|c|}
\hline Quarter 1 & Quarter 2 & Quarter 3 & Quarter 4 & YTD Reduction \\
\hline $1,026.00 \mathrm{~m} 3$ & $1,026.00 \mathrm{~m} 3$ & $1,026.00 \mathrm{~m} 3$ & $1,300.00 \mathrm{~m} 3$ & $4,378.00 \mathrm{~m} 3$ \\
\hline \multicolumn{5}{|c|}{ Savings } \\
\hline Quarter 1 & Quarter 2 & Quarter 3 & Quarter 4 & YTD Savings \\
\hline $1,500,437.00$ & $1,500,437.00$ & $1,500,437.00$ & $807,160.00$ & $5,308,471.00$ \\
\hline
\end{tabular}

Accomplishment: The herbicide spraying program for tumbleweeds on the Hanford Site encompasses 1350 acres of land identified as "Underground Radioactive Material". Spraying has proven to be the most cost effective and efficient means in controlling the spread of radioactively contaminated weeds. The other preferred option is to remove the weeds using hand labor.

\section{Hanford Site}

$\begin{array}{lllll}\text { P2 Activity } & \text { Waste Type } & \begin{array}{l}\text { Routine or } \\ \text { Cleanup/Stabilization }\end{array} & \begin{array}{l}\text { Ongoing Project or } \\ \text { New Project }\end{array} & \begin{array}{l}\text { Ongoing Project } \\ \text { Life (in years) }\end{array} \\ \begin{array}{llll}\text { Source } \\ \text { Reduction }\end{array} & \text { SAN } & \text { Cleanup/Stabilization } & \text { Ongoing } & 1\end{array}$

This accomplishment counts toward the DOE-wide reduction goal (i.e. 10\%) for waste resulting from cleanup, stabilization, and decommissioning activities.

Waste Reduction 


\begin{tabular}{|l|l|l|l|l|}
\hline Quarter 1 & Quarter 2 & Quarter 3 & Quarter 4 & YTD Reduction \\
\hline \hline $0.34 \mathrm{mt}$ & $0.00 \mathrm{mt}$ & $0.00 \mathrm{mt}$ & $0.00 \mathrm{mt}$ & $0.34 \mathrm{mt}$ \\
\hline
\end{tabular}

Savings

\begin{tabular}{|l|l|l|l||}
\hline Quarter 1 & Quarter 2 & Quarter 3 & Quarter 4 \\
\hline 70.00 & 0.00 & 0.00 & YTD Savings \\
\hline
\end{tabular}

Accomplishment: The amount of paperwork required to be generated for the necessary shipping papers of waste slated for Environmental Restoration Disposal Facility was reduced in half to only 1 sheet. Also reduced is the quantity of records that need to be stored.

\section{Hanford Site}

$\begin{array}{lllll}\text { P2 Activity } & \text { Waste Type } & \begin{array}{l}\text { Routine or } \\ \text { Cleanup/Stabilization }\end{array} & \begin{array}{l}\text { Ongoing Project or } \\ \text { New Project }\end{array} & \begin{array}{l}\text { Ongoing Project } \\ \text { Life (in years) }\end{array} \\ \begin{array}{l}\text { Source } \\ \text { Reduction }\end{array} & \text { WW-MLLW } & \text { Cleanup/Stabilization } & \text { Ongoing } & 1\end{array}$

This accomplishment counts toward the DOE-wide reduction goal (i.e. 10\%) for waste resulting from cleanup, stabilization, and decommissioning activities.

Waste Reduction

\begin{tabular}{|c|c|c|c|c|}
\hline Quarter 1 & Quarter 2 & Quarter 3 & Quarter 4 & YTD Reduction \\
\hline $0.00 \mathrm{~m} 3$ & $229.00 \mathrm{~m} 3$ & $518.00 \mathrm{~m} 3$ & $296.60 \mathrm{~m} 3$ & $1,043.60 \mathrm{~m} 3$ \\
\hline \multicolumn{5}{|c|}{$\begin{array}{c}\text { Savings } \\
\end{array}$} \\
\hline Quarter 1 & Quarter 2 & Quarter 3 & Quarter 4 & YTD Savings \\
\hline 0.00 & $15,550.00$ & $33,065.00$ & $7,841.00$ & $56,456.00$ \\
\hline
\end{tabular}

Accomplishment: On March 25, 1999 the Environmental Protection Agency signed an amendment to the Environmental Restoration Disposal Facility Record of Decision, making the Environmental Restoration Disposal Facility leachate non-hazardous. The previously mixed low level leachate collected from Environmental Restoration Disposal Facility was recycled and used as dust suppression. 


\section{HNF-6039 \\ Rev. 0 \\ DISTRIBUTION}

\section{Number of Copies}

ONSITE

13

U.S. Department of Energy, Richland Operations Office M. A Barnard
A. V. Beard
H. E. Bilson
A2-15
V. F. Boston
$\mathrm{H} 0-12$
E. M. Bowers
A6-38
M. E. Burandt
A2-15
D. H. Chapin
$\mathrm{K} 8-50$
M. S. Collins
R3-79
G. Ellis-Balone
$\mathrm{H} 0-12$
R. S. Mercado
A5-58
J. H. Zeisloft Jr.
A2-15
Hanford Technical Library
A2-15
Public Reading Room
P8-55
$\mathrm{H} 2-53$

H6-60

U.S. Department of Energy, Office of River Protection

G. M. Neath

H6-60

40

Fluor Project Hanford

D. J. Alexander

S3-30

E. S. Aromi

H6-10

B. A. Austin

H8-67

B. M. Barnes

T4-04

J. M. Bishop

L6-26

H. C. Boynton

T4-52

N. C. Boyter

L5-66

J. E. Bramson

T5-54

J. B. Buckley

T4-05

F. J. Carvo

L1-03

J. G. Coenenberg

T4-04

G. E. Entrop

T5-54

R. G. Gant

X3-79

C. K. Girres

T3.01

C. J. Grando

G3-26

B. Griffin II

T6-30

C. R. Haas

T3-28

K. A. Hadley

R3-56

T. J. Harper

H8-68 
Fluor Project Hanford, cont'd
A. E. Hill
N2-57
N. O. Hinojosa
X3-67
J. E. Hodgson
T3-04
G. S. Hunacek
X3-79
L. D. Kamberg
T4-04
G. J. LeBaron
S4-49
P. W. Martin
T3-04
D. H. Nichols
A0-25
B. G. Place
A0-25
D. L. Renberger
H6-10
P. Segall
A0-25
R. W. Szelmeczka
S6-72
J. E. Turnbaugh
X3-79
L. O. Waggoner
S0-19
G. J. Warwick
S3-25
D. J. Watson
X3-79
J. D. Williams
H8-67
J. A. Winterhalder
T6-16
Document Processing Center (1) H6-08
Central Files (1)
B1-07
Correspondence Control (1)
A3-01

2

Bechtel Hanford Inc.

D. K. Duvon

$\mathrm{HO}-02$

R. J. Landon

$\mathrm{H} 0-02$

Fluor Project Hanford Northwest

R. L. Newell

B4-68

Hanford Environmental Health Foundation

1

S. M. McInturff

$\mathrm{H} 1-77$

4

River Protection Project

D. E. Clark

R1-51

T. A. Dillhoff

S5-03

W. T. Dixon

R1-51

P. C. Miller

R1-51

1

Lockheed Martin Services, Inc.

T. M. Holmes, Jr.

G3-60

Pacific Northwest National Laboratory

1

E. V. Alderson

P7-79

Waste Management Technical Services

D. L. McCall 